\title{
VLA Radio Observations of the HST Frontier Fields Cluster Abell 2744: The Discovery of New Radio Relics
}

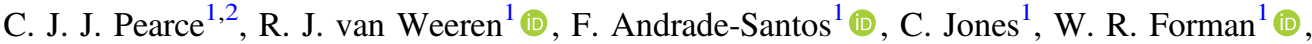 \\ M. Brüggen ${ }^{3}$, E. Bulbul ${ }^{4}$, T. E. Clarke ${ }^{5}$ (1) , R. P. Kraft ${ }^{1}$ (i), E. Medezinski ${ }^{6}$ (1),

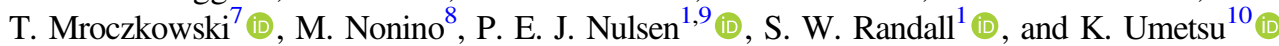 \\ ${ }^{1}$ Harvard-Smithsonian Center for Astrophysics, 60 Garden Street, Cambridge, MA 02138, USA; connorjjpearce@ gmail.com \\ ${ }^{2}$ School of Physics and Astronomy, University of Southampton, Highfield, Southampton SO17 1BJ, UK \\ ${ }^{3}$ Hamburger Sternwarte, Universität Hamburg, Gojenbergsweg 112, D-21029 Hamburg, Germany \\ ${ }^{4}$ Kavli Institute for Astrophysics and Space Research, Massachusetts Institute of Technology, 77 Massachusetts Avenue, Cambridge, MA 02139, USA \\ 5 U.S. Naval Research Laboratory, Remote Sensing Division, 4555 Overlook Avenue SW, Washington, DC 20375, USA \\ ${ }^{6}$ Department of Astrophysical Sciences, Princeton University, Princeton, NJ 08544, USA \\ ${ }^{7}$ European Organization for Astronomical Research in the Southern Hemisphere, Karl-Schwarzschild-Str. 2, D-85748 Garching b. München, Germany \\ ${ }^{8}$ INAF-Trieste Astronomical Observatory, via Bazzoni 2, I-34124 Trieste, Italy \\ ${ }^{9}$ ICRAR, University of Western Australia, 35 Stirling Highway, Crawley, WA 6009, Australia \\ ${ }^{10}$ Institute of Astronomy and Astrophysics, Academia Sinica, PO Box 23-141, Taipei 10617, Taiwan \\ Received 2017 April 5; revised 2017 June 16; accepted 2017 July 2; published 2017 August 14
}

\begin{abstract}
Cluster mergers leave distinct signatures in the intracluster medium (ICM) in the form of shocks and diffuse cluster radio sources that provide evidence for the acceleration of relativistic particles. However, the physics of particle acceleration in the ICM is still not fully understood. Here we present new 1-4 GHz Jansky Very Large Array (VLA) and archival Chandra observations of the HST Frontier Fields Cluster Abell 2744. In our new VLA images, we detect the previously known $\sim 2.1 \mathrm{Mpc}$ radio halo and $\sim 1.5 \mathrm{Mpc}$ radio relic. We carry out a radio spectral analysis from which we determine the relic's injection spectral index to be $\alpha_{\text {inj }}=-1.12 \pm 0.19$. This corresponds to a shock Mach number of $\mathcal{M}=2.05_{-0.19}^{+0.31}$ under the assumption of diffusive shock acceleration. We also find evidence for spectral steepening in the post-shock region. We do not find evidence for a significant correlation between the radio halo's spectral index and ICM temperature. In addition, we observe three new polarized diffuse sources and determine two of these to be newly discovered giant radio relics. These two relics are located in the southeastern and northwestern outskirts of the cluster. The corresponding integrated spectral indices measure $-1.81 \pm 0.26$ and $-0.63 \pm 0.21$ for the SE and NW relics, respectively. From an X-ray surface brightness profile we also detect a possible density jump of $R=1.39_{-0.22}^{+0.34}$ co-located with the newly discovered SE relic. This density jump would correspond to a shock front Mach number of $\mathcal{M}=1.26_{-0.15}^{+0.25}$.
\end{abstract}

Key words: galaxies: clusters: individual (Abell 2744) - galaxies: clusters: intracluster medium radiation mechanisms: non-thermal - X-rays: galaxies: clusters

\section{Introduction}

In the standard $\Lambda \mathrm{CDM}$ cosmological model, galaxy clusters form via a hierarchical sequence of merging events of smaller structures, growing from small groups of galaxies to major clusters. Cluster mergers, driven by the gravitational interaction of the dominant dark matter, can release up to $10^{63}-10^{64} \mathrm{erg}$ of energy on timescales of about a gigayear (one cluster crossing time). Shocks are driven into the intracluster medium (ICM) during such mergers, which dissipate the energy into heating the gas, along with the subsequent turbulent ICM motions that follow.

Part of the energy that is dissipated during these collisions could be fed into (re)accelerating relativistic particles and amplifying the ICM magnetic field, resulting in cluster-scale diffuse synchrotron radiation. At radio wavelengths we see evidence of this acceleration in the form of radio halos and radio relics (for reviews see Feretti et al. 2012; Brunetti \& Jones 2014).

Radio halos are large ( $\gtrsim 1 \mathrm{Mpc}$ ) diffuse radio sources located in the center of a cluster. They have a smooth regular morphology and are unpolarized down to a level of a few per cent. Their emission typically follows the observed thermal $\mathrm{X}$-ray emission and has a steep spectrum $(\alpha \lesssim-1)$. Radio relics are irregularly shaped sources of a similar scale to halos $(\sim 0.5-2.0 \mathrm{Mpc})$, but are located in the cluster periphery and polarized at the $10 \%-60 \%$ level, indicating the presence of ordered magnetic fields. Like halos, they also have steep synchrotron spectra. A strong correlation between cluster-scale synchrotron emission and cluster mergers is observed due to the fact that these radio sources are preferentially found in dynamically disturbed systems (Cassano 2010). The exact origin of the radio emission in these features is still being debated. The main problem is reconciling the relatively short radiative lifetime of the electron $\left(\sim 10^{8} \mathrm{yr}\right)$ with the megaparsec size and roughly gigayear age of these sources. Therefore some form of in situ acceleration process must be occurring (Jaffe 1977).

For radio halos the current theories are that of turbulent reacceleration (primary models, Brunetti et al. 2001; Petrosian 2001), and the production of secondary electrons during collisions between thermal ICM protons and cosmic-ray protons trapped in the ICM (secondary models, Dennison 1980).

Radio relics are subdivided into three categories: giant radio relics, relics of active galactic nuclei (AGNs), and radio phoenices (Kempner et al. 2004). Giant radio relics are arc-like sources of synchrotron radiation of megaparsec size. The leading theory behind their formation is that of shock acceleration (Enßlin et al. 1998; Drury 1983) of either thermal or preaccelerated fossil electrons from AGNs or other radio 
galaxy activity (e.g., Markevitch et al. 2005; Giacintucci et al. 2008; Kang \& Ryu 2011; Pinzke et al. 2013; van Weeren et al. 2017a), although other models exist (Fujita et al. 2015).

Radio phoenices are also believed to be caused by shocks. However, in this case the shocks only adiabatically compress fossil relativistic plasma from AGNs (Enßlin \& Krishna-Gopal 2001; van Weeren et al. 2011; de Gasperin et al. 2015). AGN relics, on the other hand, are regions/lobes of fossil plasma from radio galaxies without any reacceleration/compression having occurred.

In this paper we present a radio spectral analysis of the merging cluster Abell 2744 from Jansky Very Large Array (VLA) observations at 1-4 GHz. With these observations we study the nature of the diffuse radio emission in this cluster. In Section 2 we provide an overview of Abell 2744, describing the results of previous X-ray, radio, and optical studies. In Section 3 we explain the calibration and reduction processes used for both the X-ray and radio data. In Section 4 we present the radio maps constructed at various resolutions along with the associated spectral index maps. Integrated radio spectra and a polarization vector map are also shown. In Section 5 we discuss the application of our results to the currently proposed theories of synchrotron acceleration. We extract radial profiles of the spectral index across both the radio relic and halo, and perform an analysis of the variation in spectral index across the radio halo. Three previously unobserved diffuse sources are discussed in more detail. The results are summarized in Section 6.

Throughout this paper we assume a flat, $\Lambda \mathrm{CDM}$ cosmology with $H_{0}=70 \mathrm{~km} \mathrm{~s}^{-1} \mathrm{Mpc}^{-1}$, matter density $\Omega_{m}=0.3$, and dark energy density $\Omega_{\Lambda}=0.7$ (Bennett et al. 2014). At the cluster's redshift, $1^{\prime}$ corresponds to a scale of $\sim 272 \mathrm{kpc}$.

Cluster, foreground, and lensed compact radio sources will be discussed in detail in a separate paper.

\section{Abell 2744}

Abell 2744 is a complex merger event located at $z=0.308$. Owing to its virial mass and large area of high magnification, it was chosen as one of the HST Frontier Fields clusters (Lotz et al. 2014, 2017).

X-ray observations have revealed several substructures near the center, including cold and dense remnant gas cores to the north and south, a prominent hot gas cloud between the two main galaxy groups, as well as an additional X-ray-luminous structure to the northwest (Kempner \& David 2004). Kinematic studies also showed a bimodal velocity dispersion in the cluster center, with a third group of cluster members associated with the northwestern X-ray peak (Boschin et al. 2006; Braglia et al. 2009).

The merger scenario for this cluster is that of a primary, bulletlike merger event (Markevitch et al. 2002) that took place in the $\mathrm{N}-\mathrm{S}$ direction with a large line-of-sight (LOS) component to the merger axis, and that a secondary event is occurring with the infall of a third subcluster (Kempner \& David 2004). From $\mathrm{X}$-ray analysis it was suggested that the merger mass ratio of the subclusters is roughly equal (Kempner \& David 2004), though it was unclear whether the dominant massive cluster core was located to the south (Owers et al. 2011) or the north (Kempner \& David 2004). The role of the northwest subcluster is also debated, with recent analysis of Chandra data by Owers et al. (2011) suggesting that it is actually likely in a post, off-center, core passage phase heading toward the north/northeast.

A more complicated explanation was suggested by Merten et al. (2011), who conducted a detailed gravitational lensing analysis of the cluster. They identify four distinct substructures corresponding to peaks in surface mass density, including a prominent southern core, a northern clump, a northwestern clump of dark matter separated from the known interloper gas cloud, and a western "Ghost" substructure completely stripped of gas. In order to explain the positional offset of the dark matter peaks and $\mathrm{X}$-ray brightness peaks, they suggest a complex merger scenario in which an initial NE-SW merger caused a "slingshot" effect in a second, almost simultaneous NW-SE merger event (see Figure 9 of Merten et al. 2011).

More recently, a weak-lensing analysis of A2744 was conducted by Medezinski et al. (2016) using new Subaru/Suprime-Cam images. They identified four substructures similar to those of Merten et al. (2011) but determined a much reduced mass for the northern core, and instead identified a more prominent northeastern core. They therefore proposed that a major merger event occurred in the east-west direction with another taking place in the northsouth direction just east of center, which pushed the main cluster gas toward the northwest. Based on multiple $N$-body simulations they find the "slingshot" scenario offered by Merten et al. (2011) unlikely. Instead, based on the observation of two associated dark matter peaks, they suggest that the interloper is the result of a third, minor off-axis merger event between two subhalos close to the LOS. During this event the gas from both subhalos was completely stripped, resulting in the observed separations in dark matter and X-ray luminosity peaks. Interestingly, in another recent lensing analysis, Jauzac et al. (2016) detected an additional four substructures, further complicating the picture, see Figure 1.

\subsection{Previous Radio Studies of Abell 2744}

Radio emission in Abell 2744 was originally identified by Giovannini et al. (1999), who detected a peripheral radio relic to the northeast and a central radio halo. The cluster has since been observed at radio frequencies of $1.4 \mathrm{GHz}$ by Govoni et al. (2001) and at $325 \mathrm{MHz}$ by Orrú et al. (2007) and Venturi et al. (2013). Herein these observations shall be referred to as "G01," "O07," and "V13" respectively. From the $1.4 \mathrm{GHz}$ observations, Govoni et al. (2001) determined the halo to be one of the most powerful known $\left(P_{1.4 \mathrm{GHz}} \approx 2.6 \times 10^{25} \mathrm{~W} \mathrm{~Hz}^{-1}\right)$.

In the investigation by $\mathrm{O} 07$, the radio halo was found to have a patchy spectral index distribution in accordance with primary models (e.g., Ferrari et al. 2008). A spatial correlation was also suggested between the halo regions with flattest spectral indices and those with the highest X-ray temperature. Hints of a spectral index gradient across the relic in A2744 were identified by $\mathrm{O} 07$, with spectral steepening occurring inward in the direction of the cluster center. Such gradients across relics are common and are thought to be the result of energy losses in the downstream post-shock region (e.g., van Weeren et al. 2010). Ibaraki et al. (2014) found evidence of an X-ray shock in the form of a temperature jump, co-located with the known radio relic (R1, see Figure 1) in A2744. More recently, using XMM-Newton and Suzaku data, Eckert et al. (2016) reported the detection of a jump in surface brightness and temperature at the eastern edge of the $\mathrm{R} 1$ radio relic, corresponding to a weak shock with a Mach number of $\mathcal{M}=1.7_{-0.3}^{+0.5}$ (1 $\sigma$ uncertainties).

\section{Observations and Data Reduction}

\subsection{VLA Observations}

A2744 was observed with the VLA in the 1-2 GHz L-band and $2-4 \mathrm{GHz}$ S-band in the $\mathrm{DnC}-, \mathrm{CnB}-$, and BnA-array 


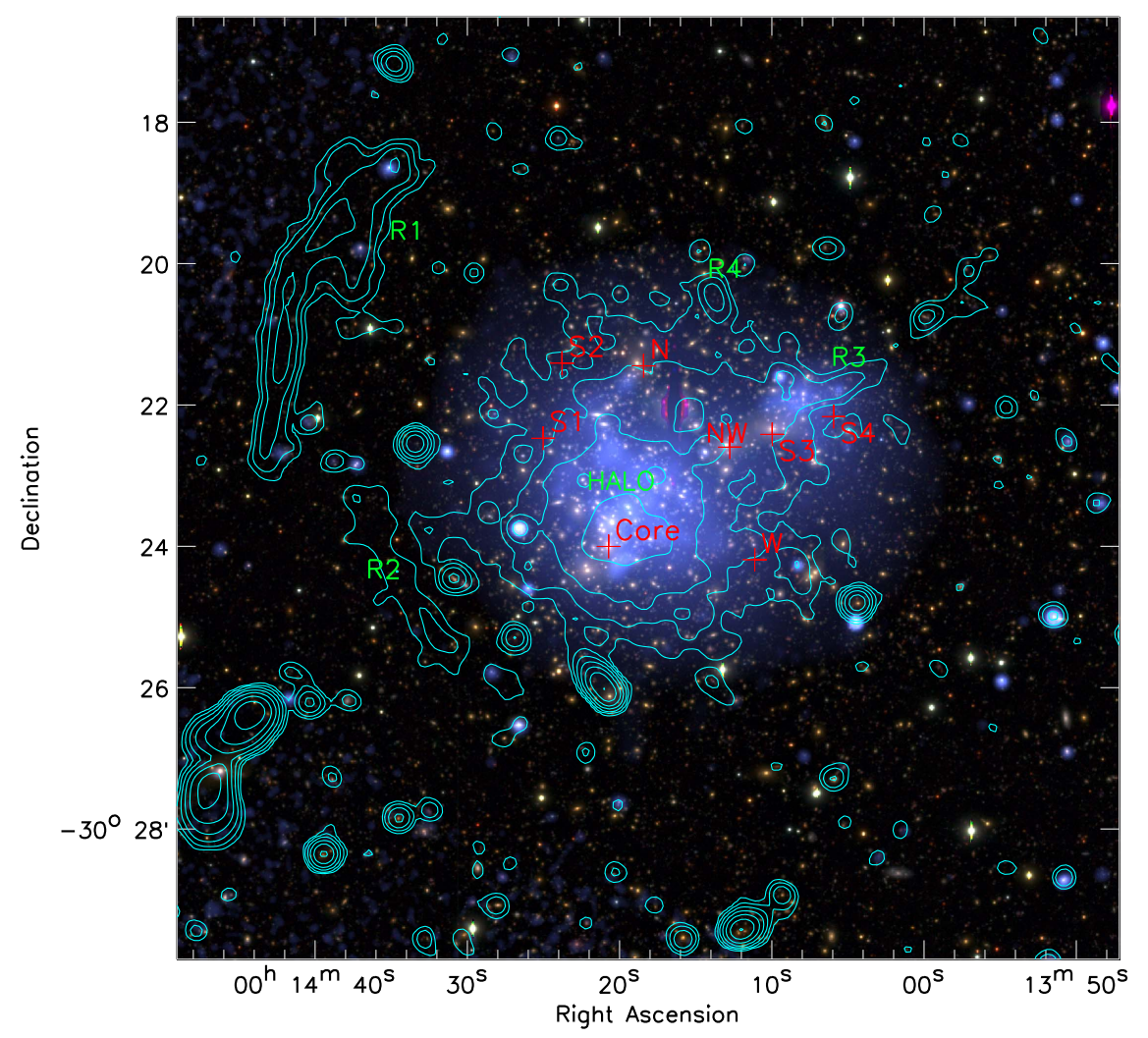

Figure 1. Optical, X-ray, and radio overlay of A2744. The Subaru optical $B R z$ color image comes from Medezinski et al. (2016). The Chandra 0.5-2.0 keV image is shown in blue. Overlaid in cyan are radio contours from the $1-2 \mathrm{GHz}$ wideband $15^{\prime \prime}$ uv-tapered map. The contour levels are drawn at $[1,2,4, \ldots] \times 5 \sigma_{\mathrm{rms}}$. Cluster substructures are labelled with red crosses following Jauzac et al. (2016). Diffuse extended cluster radio sources are indicated with green labels; see also Figure 5.

configurations. An overview of the observations is given in Tables 1 and 2. The data were recorded with the default wideband setup of 16 spectral windows spanning the entire bandwidth, with each window having 64 channels.

The data have been calibrated using the Common Astronomy Software Applications (CASA; McMullin et al. 2007) package version 4.4.0. First the data were Hanning-smoothed and data affected by RFI (radio-frequency interference) and other sources such as antenna shadowing were flagged. The predetermined elevation-dependent gain tables and antenna offset positions were also applied. An initial set of gain solutions was determined for the primary calibrator sources $3 \mathrm{C} 147$ and $3 \mathrm{C} 138$ over a small window of channels in the center of the bandpass where the phase variations per channel are small. This removes any timedependent effects of phase variation in each channel. We then calibrated the delays and bandpass in conjunction with the gain solutions. Next, the cross-hand delays were calibrated using the polarized calibrator $3 \mathrm{C} 138$. The gain solutions for the phase calibrator (J0011-2612) were determined along with the polarization leakages. The gains for all the calibrators were then combined, solving for the J0011-2612 flux density. We used the flux scale of Perley \& Butler (2013). As a final step all relevant calibration solutions were applied to the target field data (A2744). After initial calibration and flagging, several rounds of selfcalibration were performed to further refine the calibration for each individual data set. All images were made employing the W-projection algorithm in CASA (Cornwell 2008). Clean masks were used for each image step. These masks were made using the PyBDSM source detection package (Mohan \& Rafferty 2015). The spectral index and curvature were taken into account during the deconvolution (nterms = 3; Rau \& Cornwell 2011).
Table 1

L-band Observations

\begin{tabular}{llll}
\hline \hline & DnC Array & CnB Array & BnA Array \\
\hline Observation dates & 2014 Sep 22 & 2015 Jan 10 & 2015 June 2 \\
$\begin{array}{l}\text { Total on-target observing } \\
\quad \text { time (hr) }\end{array}$ & 2.3 & 5 & 5 \\
$\begin{array}{l}\text { Frequency range (GHz) } \\
\text { Correlations }\end{array}$ & $1-2$ & $1-2$ & $1-2$ \\
Largest angular scale (arcsec) & full Stokes & full Stokes & full Stokes \\
Channel width (MHz) & 1 & 970 & 120 \\
& & 1 & 1
\end{tabular}

After self-calibration, the data sets were combined (in each relative frequency band). We imaged the combined data sets using WSClean (Offringa et al. 2014), using Briggs weighting (Briggs 1995) with robust $=0.0$ and employing the wideband and multiscale algorithms and using various uv tapers. Again, clean masks were constructed as mentioned above. The images were corrected for the primary beam attenuation using primary beam images created in CASA.

In order to create spectral index maps we imaged the combined data sets in CASA with an inner uv range cut (corresponding to the shortest baselines in the S-band data), uniform weighting, Gaussian taper, and multiscale clean (Rau \& Cornwell 2011). Each data set was imaged four times, each tapered to a different resolution in order to resolve diffuse emission on varying spatial scales. The final images were then corrected for primary beam attenuation.

To create the deepest possible radio maps we imaged the Land S-band data sets together using WSClean. These images were only used as visual guides and no flux density values quoted in 
Table 2

S-band Observations

\begin{tabular}{llll}
\hline \hline & DnC Array & CnB Array & BnA Array \\
\hline Observation dates & 2014 Sep 20 & Run 1: 2015 Jan 11 & Run 1: 2015 May 22 \\
& & Run 2: 2015 Jan 13 & Run 2: 2015 May 23 \\
Total on-target observing time (hr) & 2.3 & $4(2+2)$ & $10(5+5)$ \\
Frequency range (GHz) & $2-4$ & $2-4$ & $2-4$ \\
Correlations & full Stokes & full Stokes & full Stokes \\
Largest angular scale (arcsec) & 490 & 490 & 58 \\
Channel width $(\mathrm{MHz})$ & 2 & 2 & 2 \\
\hline
\end{tabular}

Table 3

Image Properties

\begin{tabular}{|c|c|c|c|}
\hline Image & Weighting & Resolution $(\operatorname{arcsec} \times \operatorname{arcsec})$ & Rms Noise Level $\left(\mu \mathrm{Jy}\right.$ beam $\left.^{-1}\right)$ \\
\hline L-band uv taper $30^{\prime \prime}$ & Uniform & $30 \times 30$ & 31 \\
\hline L-band uv taper $15^{\prime \prime}$ & Uniform & $15 \times 15$ & 19 \\
\hline L-band uv taper $10^{\prime \prime}$ & Uniform & $10 \times 10$ & 16 \\
\hline L-band & Briggs, robust $=0.0$ & $4.15 \times 2.83$ & 10 \\
\hline S-band uv taper $30^{\prime \prime}$ & Uniform & $30 \times 30$ & 43 \\
\hline S-band uv taper $15^{\prime \prime}$ & Uniform & $15 \times 15$ & 15 \\
\hline S-band & Briggs, robust $=0.0$ & $1.65 \times 1.40$ & 4.1 \\
\hline $1-4 \mathrm{GHz}$ wideband uv taper $30^{\prime \prime}$ & Briggs, robust $=0.0$ & $30.5 \times 29.8$ & $\ldots$ \\
\hline $1-4 \mathrm{GHz}$ wideband uv taper $15^{\prime \prime}$ & Briggs, robust $=0.0$ & $15.6 \times 15.3$ & $\ldots$ \\
\hline $1-4 \mathrm{GHz}$ wideband uv taper $10^{\prime \prime}$ & Briggs, robust $=0.0$ & $10.9 \times 10.4$ & $\cdots$ \\
\hline $1-4 \mathrm{GHz}$ wideband uv taper $5^{\prime \prime}$ & Briggs, robust $=0.0$ & $6.01 \times 5.52$ & $\cdots$ \\
\hline 1-4 GHz wideband & Briggs, robust $=0.0$ & $2.29 \times 1.81$ & $\ldots$ \\
\hline
\end{tabular}

this paper have been extracted from them. A summary of the final image properties is given in Table 3. The S-band polarization image will be discussed in Section 4.5.

\subsection{Chandra Observations}

We used $125 \mathrm{ks}$ of archival Chandra ACIS observations (ObsID: 7712, 2212, 7915, 8477, 8557). As described in Vikhlinin et al. (2005), the data were calibrated using the chav package, applying the most recent calibration files. ${ }^{11}$ The calibration process involves filtering all counts from bad pixels and counts with recomputed ASCA grade of 1,5 , or 7 . We then corrected for position-dependent charge transfer inefficiency and applied gain maps to calibrate photon energies. Periods of high background during the observation time were also filtered out by examining the count rate in the $6-12 \mathrm{keV}$ band and removing periods with a flux 1.2 times above the mean. The total filtered clean exposure time was $126 \mathrm{ks}$. Standard blank sky background files were used for background subtraction. We used a pixel binning factor of 4 . For more details about the data reduction the reader is referred to Vikhlinin et al. (2005).

\section{Results}

In Figures 2-4 we present the combined L- and S-band total intensity radio maps at different resolutions. See Figure 5 for the labelling of sources. At all resolutions we detect both the radio halo and the relic (R1), along with several other radio sources. In Figure 6 we display a radio and X-ray overlay of the cluster. A combined X-ray and optical image, marked with the subcluster

$\overline{11}$ We used CIAO v4.8 and CALDB v4.7.2. components found by Jauzac et al. (2016), is displayed in Figure 1.

From the low-resolution VLA images we find that the halo has an extent of $\sim 2.1 \mathrm{Mpc}$ and that it roughly follows the $\mathrm{X}$-ray emission from the ICM (see Figure 6). The peak radio emission in the central part of the halo also appears to be colocated with the peak X-ray emission. The morphology of the halo is similar to that observed by O07, V13, and G01, with a slight elongation in the NW direction and an asymmetric brightness distribution. The largest linear size (LLS) we detect of 2.1 Mpc appears to be a good average of the values of 1.6, 1.9, and 2.34 Mpc obtained by O07, V13, and G01.

The morphology of the relic (R1) is similar to that seen in previous observations but with some notable new features. The high-resolution images reveal that the relic does not appear to have the same smoothly curved shape as previously seen; instead we find it has a relatively straight morphology with a distinct "kink" in it. Interestingly, the upper portion of the relic appears to be further complicated by a second linear component of diffuse emission extending away from the shock front (labelled R1-A in Figure 5).

Diffuse elongated source R2, located to the south of R1, covers an area of $1.15 \times 0.25 \mathrm{Mpc}^{2}$ and is located approximately $0.9 \mathrm{Mpc}$ from the cluster center. It is best visible in the lowresolution L-band images, but also appears in the low-resolution S-band images. In addition, we observe a filamentary thin $1.1 \mathrm{Mpc}$ elongated source (R3) to the NW of the radio halo. The source seems to protrude directly outward from the northwest subcluster, which is also covered by emission from the radio halo. However, due to our line of sight it is unclear whether or not it is projected on top of the halo or if it is connected to it. Finally, we identify a patch of diffuse emission north of the radio halo (labelled R4 in 

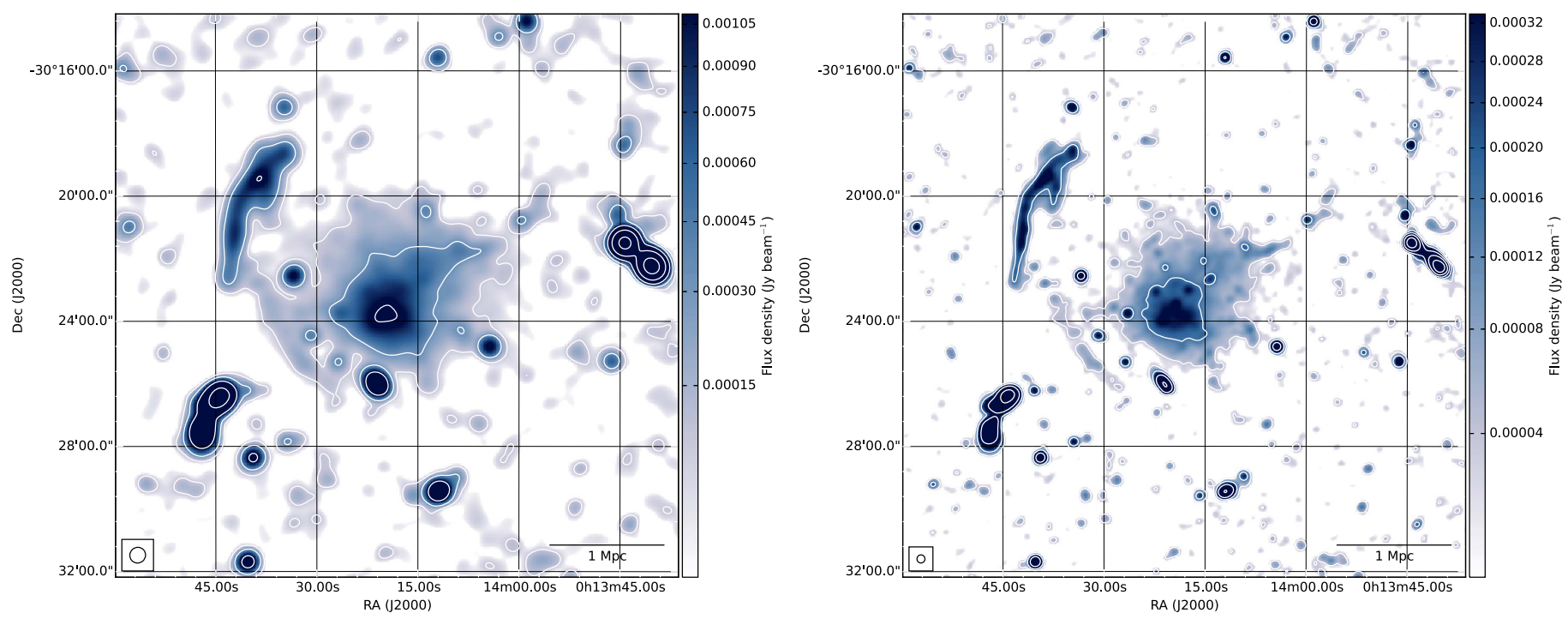

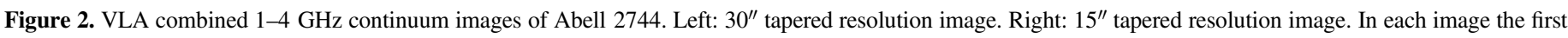
contour is at the $3.5 \sigma_{\mathrm{rms}}$ level, with additional contours spaced by factors of 4 .

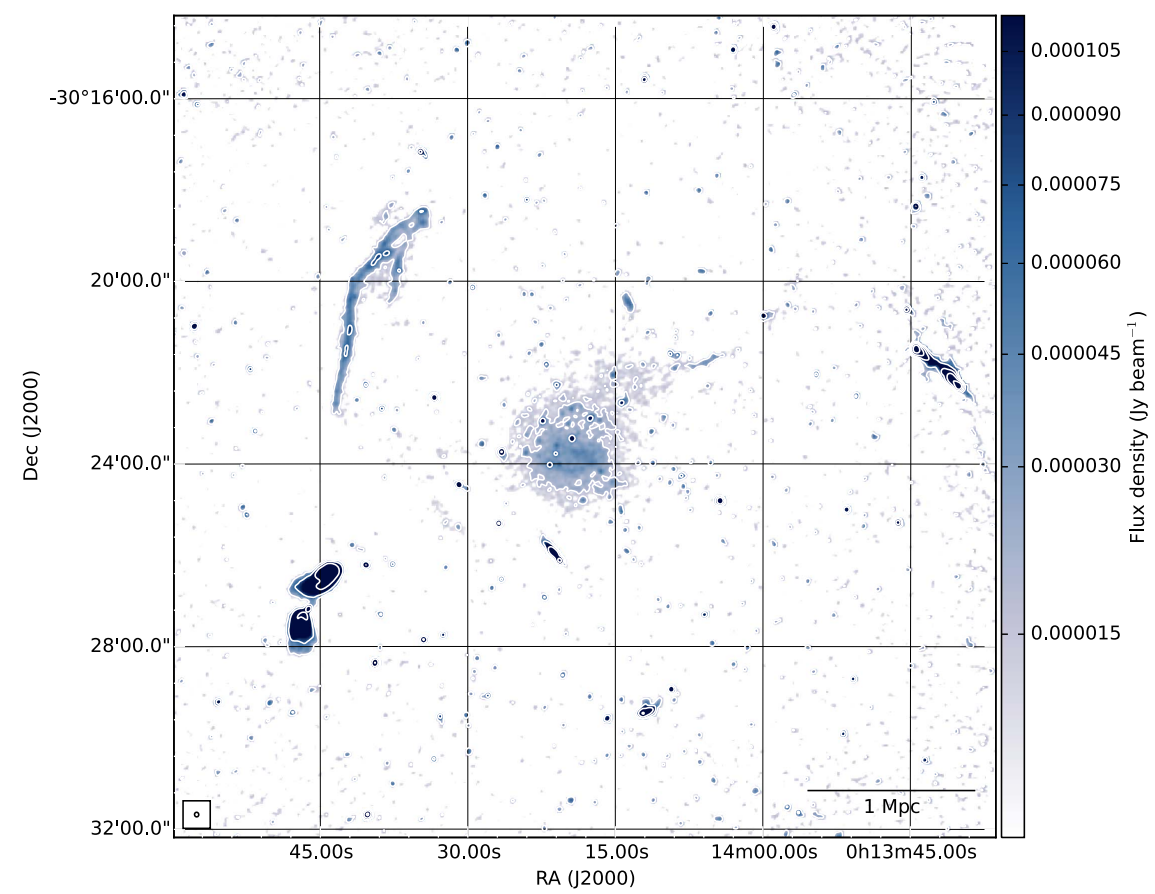

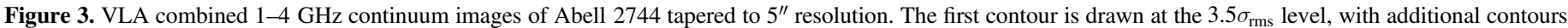
spaced by factors of 4 .

Figure 5, see also Figure 4), 200 kpc from the cluster center. It covers a much smaller area than the other diffuse sources $\left(50 \times 30 \mathrm{kpc}^{2}\right)$. We do not find an optical counterpart to the source in the images from Medezinski et al. (2016).

\subsection{Other Individual Sources}

In Figure 5 we mark several compact radio sources within the field of view. Source A is located roughly halfway between the relic and the cluster center (see Section 5.2.1). It is identified in V13 as source Abell 2744: [VGD2013] S2. Source B is the "Jellyfish" galaxy F0083 identified by Owers et al. (2012). These galaxies are characterized by trailing knots of star formation caused by extreme ram-pressure stripping (Cortese et al. 2007; Ebeling et al. 2014). Source C, known as NVSS J001421-302558, is an example of a "head-tail" radio galaxy. Source D is briefly discussed in Section 4.2.1.

The foreground source $\mathrm{E}(z=0.1966$; Colless et al. 2003) is a radio galaxy, identified in the NRAO VLA Sky Survey (NVSS) as three separate radio components-NVSS J001444302635, NVSS J001446-302722, and NVSS J001445-302644 -due to its extended nature (together making up a single radio source). In a similar fashion, source $\mathrm{F}$ is also identified as two individual components-NVSS J001340-302212 and NVSS 001344-302130.

\subsection{Integrated Fluxes}

In order to obtain accurate measurements of the integrated radio spectra of the halo and the relic, the flux densities of all compact 


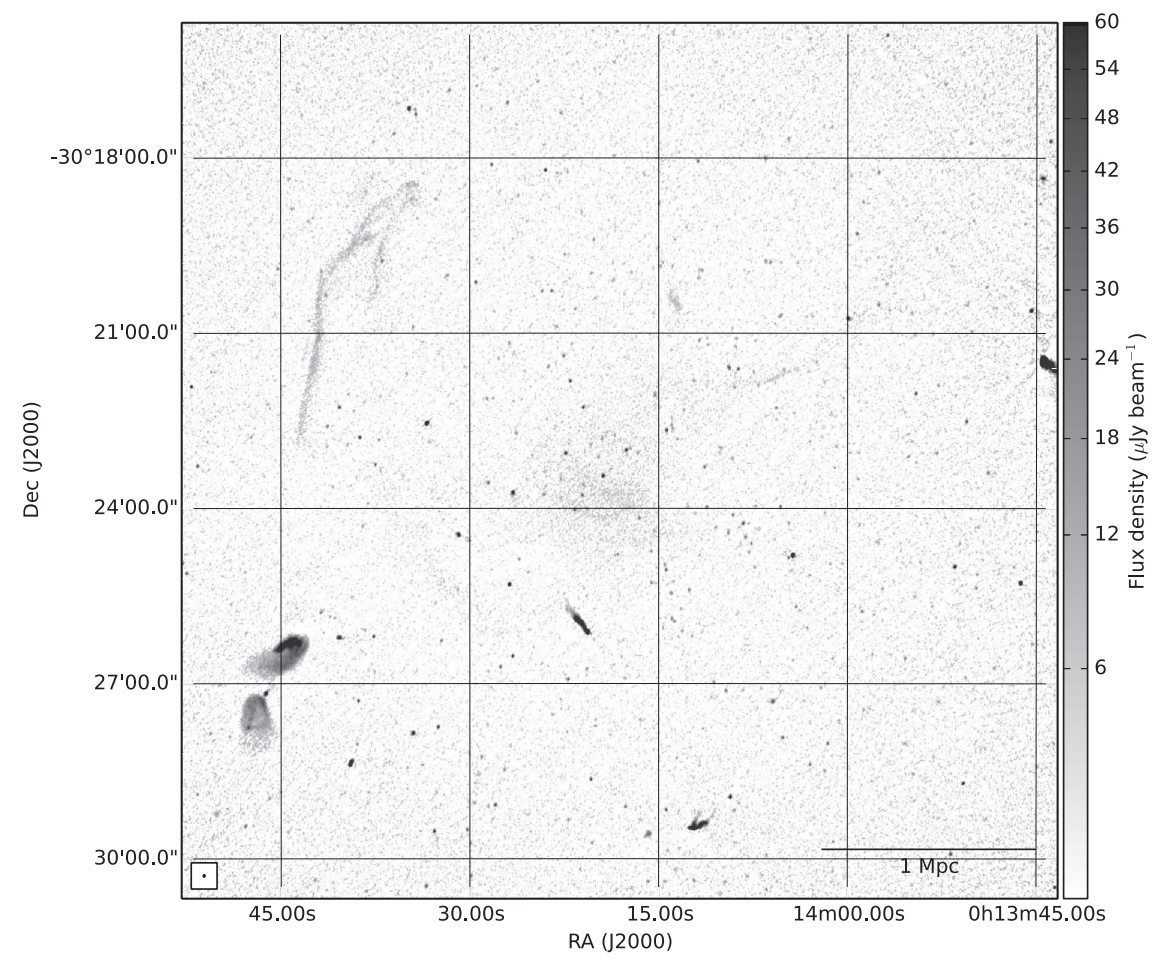

Figure 4. VLA combined 1-4 GHz high-resolution continuum image of Abell 2744 . The beam size is 2 "' $29 \times 1$ "' 81 .
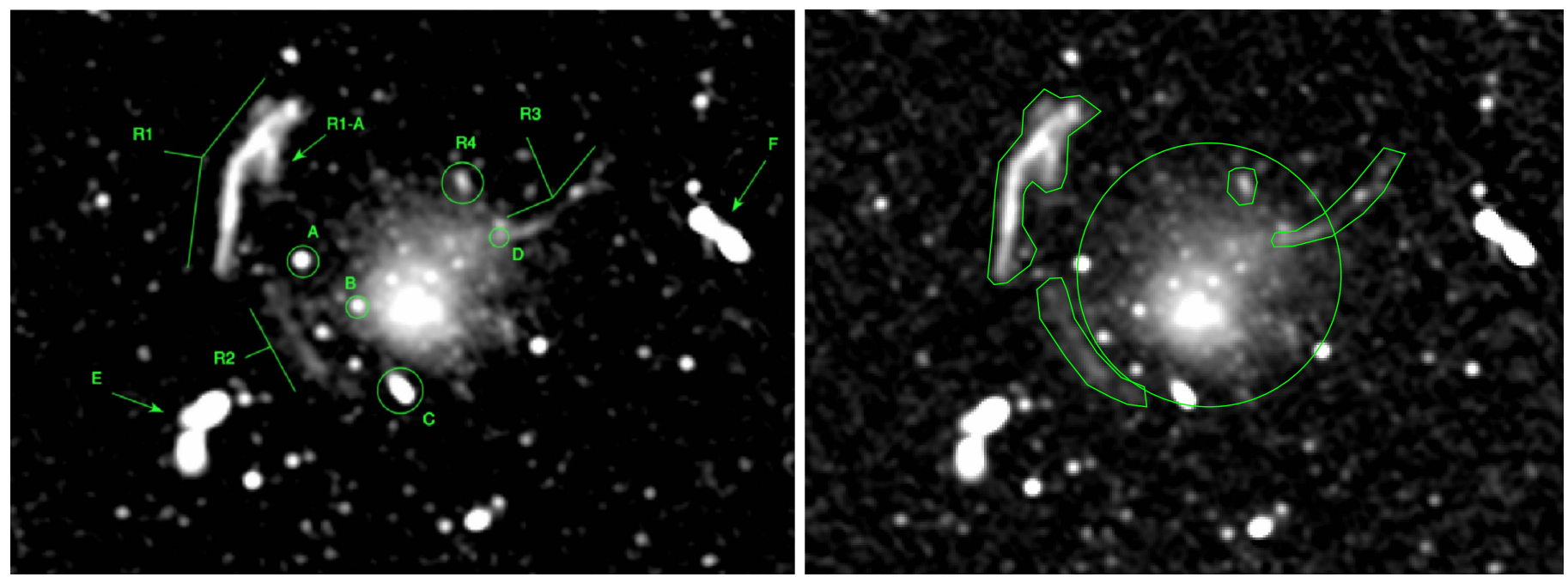

Figure 5. Left: image showing the regions of interest within the field of view that are discussed in this paper. Shown in grayscale is the L-band uv-tapered $15^{\prime \prime}$ resolution image. Right: the regions used to calculate the integrated flux densities of the diffuse cluster sources. In grayscale is the L-band uv-tapered $15^{\prime \prime}$ resolution image.

sources within the regions of interest need to be carefully subtracted from the total diffuse emission. Figure 5 (right panel) shows the regions used to calculate the integrated flux densities for the diffuse sources. Note that, unless stated otherwise, all flux density values mentioned in this section are obtained from the $15^{\prime \prime}$ resolution radio maps, where we find the highest signal-to-noise ratio.

The uncertainties $\left(\sigma_{S}\right)$ in the flux density measurements $(S)$ were taken as

$$
\sigma_{S}=\sqrt{(0.05 S)^{2}+\sigma_{R S}^{2}}
$$

where

1. $\sigma_{R S}=\sqrt{\sigma_{R}^{2}+\sigma_{C S 1}^{2}+\sigma_{C S 2}^{2}+\ldots}$ is the statistical error on the diffuse source flux density. $\sigma_{R}=\sigma_{\text {rms }} \times \sqrt{N_{\text {beams }}}$, with $\sigma_{\text {rms }}$ being the image noise level and $N_{\text {beams }}$ being the number of beams covered by the diffuse source. $\sigma_{C S}$ is the statistical error on the flux densities of the individual compact sources (where applicable), as reported by the PyBDSM software package.

2. $0.05 S$ is the error due to calibration uncertainties, taken as $5 \%$.

The measured source flux densities, spectral index values, and observed physical characteristics of the relic (R1), halo, and diffuse sources R2, R3, and R4 are summarized in Table 4. More details are given in the subsections below.

\subsubsection{Halo}

For the halo, we subtracted all point sources by imaging the field using an inner uv range cut of $3.2 k \lambda$ (robust $=0$ ), 
Table 4

Properties of Diffuse Radio Sources

\begin{tabular}{|c|c|c|c|c|c|c|c|c|c|}
\hline Source & $\begin{array}{c}D_{\perp} \\
(\mathrm{Mpc})\end{array}$ & $\begin{array}{c}\text { LLS } \\
(\mathrm{Mpc})\end{array}$ & $\begin{array}{l}S_{1.5 \mathrm{GHz}} \\
(\mathrm{mJy})\end{array}$ & $\begin{array}{l}S_{3 \mathrm{GHz}} \\
(\mathrm{mJy})\end{array}$ & $\begin{array}{c}P_{1.4 \mathrm{GHz}} \\
\left(10^{24} \mathrm{~W} \mathrm{~Hz}{ }^{-1}\right)\end{array}$ & $\alpha$ & $\alpha_{\mathrm{inj}}$ & $\mathcal{M}^{\mathrm{a}}$ & $\overline{\bar{P}^{\mathrm{b}}}$ \\
\hline Halo & $\ldots$ & 2.1 & $45.1 \pm 2.3$ & $17.0 \pm 1.0$ & $17.4 \pm 0.9$ & $-1.43 \pm 0.11$ & 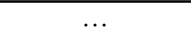 & 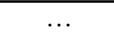 & $\bar{\ldots}$ \\
\hline Relic (R1) & 1.3 & 1.5 & $11.74 \pm 0.62$ & $4.78 \pm 0.14$ & $4.37 \pm 0.23$ & $-1.32 \pm 0.09$ & $-1.12 \pm 0.19$ & $2.05_{-0.19}^{+0.31}$ & $27 \%$ \\
\hline $\mathrm{R} 2$ & 0.9 & 1.15 & $2.18 \pm 0.17$ & $0.64 \pm 0.10$ & $0.96 \pm 0.07$ & $-1.81 \pm 0.26$ & $-1.31 \pm 0.26^{\mathrm{c}}$ & $1.86_{-0.17}^{+0.29}$ & $43 \%$ \\
\hline R3 & $\ldots$ & $\gtrsim 1.1$ & $1.46 \pm 0.14$ & $0.95 \pm 0.10$ & $0.47 \pm 0.04$ & $-0.63 \pm 0.21$ & $\ldots$ & $\cdots$ & $30 \%$ \\
\hline R4 & 0.2 & 0.05 & $0.88 \pm 0.07$ & $0.35 \pm 0.05$ & $0.30 \pm 0.03$ & $-1.34 \pm 0.23$ & $-0.84 \pm 0.23^{\mathrm{c}}$ & $2.62_{-0.50}^{+1.76}$ & $30 \%$ \\
\hline
\end{tabular}

Notes.

${ }^{a}$ Derived from $\alpha_{\text {inj }}$ and Equation (7).

b Emission-weighted mean.

c $\alpha_{\text {inj }}$ was not measured directly, but calculated using Equation (8).

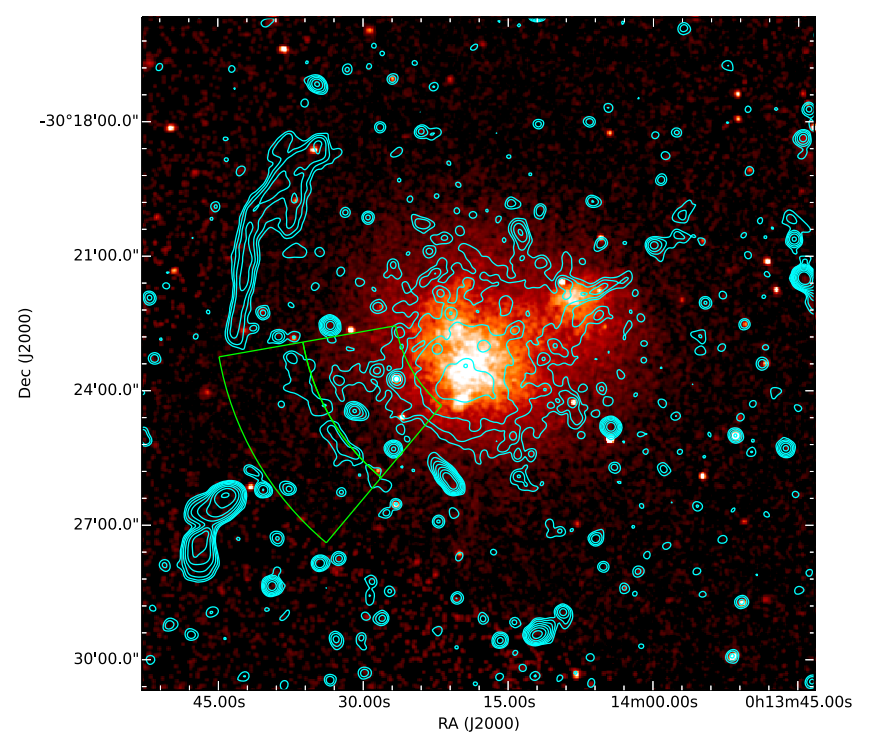

Figure 6. A2744 radio and X-ray overlay. The background-subtracted, exposure-corrected Chandra $0.5-2.0 \mathrm{keV}$ image is shown in orange. Overlaid in cyan are radio contours from the $1-4 \mathrm{GHz}$ wideband $10^{\prime \prime}$ uv-tapered radio map. The contour levels correspond to the $3.5 \sigma_{\text {rms }}$ level and above, with scaled spacings of factors of 4 . The sector used to extract a surface brightness profile from the X-ray image is displayed in green; see Section 5.2.2. The central line within the sector indicates the location of the detected density jump, at a distance of $r_{\text {edge }}=5.15_{-0.13}^{\prime+0.16}$ from the center of the sector.

corresponding to elimination of spatial scales of $\gtrsim 300 \mathrm{kpc}$. This model was then subtracted from the uv data. The data were then re-imaged with uniform weighting and a $30^{\prime \prime}$ uv taper. After this process, sources C, D, and R4 (see Figure 5) remained visible as discrete sources, predominantly in the S-band. The fluxes from these sources were manually measured and then subtracted.

After accounting for primary beam attenuation and source subtraction, we find that the radio halo has integrated flux densities of $S_{1.5 \mathrm{GHz}}=45.14 \pm 2.34 \mathrm{mJy}$ in the L-band and $S_{3 \mathrm{GHz}}=17.03 \pm 0.99 \mathrm{mJy}$ in the S-band. Using these values, we find the integrated spectral index value of the halo to be $\alpha=-1.43 \pm 0.11$.

Based upon the L-band integrated flux density measurements, the monochromatic radio power is calculated to be $P_{1.4 \mathrm{GHz}}=(1.74 \pm 0.09) \times 10^{25} \mathrm{~W} \mathrm{~Hz}^{-1}$ using the relation

$$
P_{1.4 \mathrm{GHz}}=4 \pi D_{L}^{2} S_{\nu_{0}} \nu_{0}(1+z)^{-(\alpha+1)},
$$

where $D_{L}$ is the luminosity distance to the source, $S_{\nu_{0}}$ is the integrated flux density at observing frequency $\nu_{0}(1.5 \mathrm{GHz}$ in our case), $z$ is the redshift of the source, and $\alpha$ is the spectral index used in the $k$-correction, taken as -1.43 . The radio power is in reasonable agreement with previous measurements.

\subsubsection{Relic and Other Diffuse Emission}

The same uv cut could not be applied to remove compact sources from the regions of the relic (R1), R2, R3, and R4 because these sources contained diffuse emission corresponding to physical scales smaller than $\sim 300 \mathrm{kpc}$. We used the PyBDSM source detection package to identify all compact sources in the high-resolution (robust $=0.0$ ) images with flux densities above the $5 \sigma$ level.

The total flux of the relic R1, after source subtraction of compact sources, measures $S_{1.5 \mathrm{GHz}}=11.74 \pm 0.62 \mathrm{mJy}$ in the L-band and $S_{3 \mathrm{GHz}}=4.78 \pm 0.14 \mathrm{mJy}$ in the S-band. This corresponds to an integrated spectral index value of $\alpha=$ $-1.32 \pm 0.09$. Using Equation (2) we estimate the monochromatic radio power of the relic to be $P_{1.4 \mathrm{GHz}}=(4.37 \pm$ $0.23) \times 10^{24} \mathrm{~W} \mathrm{~Hz}^{-1}$, where we have used our measured integrated spectral index value of $\alpha=-1.32$.

Diffuse source R2 has significantly lower flux densities measuring $S_{1.5 \mathrm{GHz}}=2.18 \pm 0.17 \mathrm{mJy}$ and $S_{3 \mathrm{GHz}}=0.64 \pm$ $0.10 \mathrm{mJy}$. The average spectral index value is steeper than R1 with $\alpha=-1.81 \pm 0.26$. In contrast, source R3 to the NW of the halo has a flatter spectrum. The integrated flux density values of $S_{1.5 \mathrm{GHz}}=1.46 \pm 0.14 \mathrm{mJy}$ and $S_{3 \mathrm{GHz}}=0.95 \pm 0.10 \mathrm{mJy}$ correspond to a spectral index value of $\alpha=-0.63 \pm 0.21$. For R4, we find flux densities of $S_{1.5 \mathrm{GHz}}=0.88 \pm 0.07 \mathrm{mJy}$ and $S_{3 \mathrm{GHz}}=0.35 \pm 0.05 \mathrm{mJy}$, which correspond to a spectral index of $\alpha=-1.34 \pm 0.23$.

\subsection{Integrated Radio Spectrum Using P-band Data}

Using the observed flux densities, we produced integrated radio spectra for the relic (R1) and the halo by combining our measured flux densities at both frequencies $(1.5 \mathrm{GHz}$ and $3.0 \mathrm{GHz})$ with those obtained in the P-band at $325 \mathrm{MHz}$ by 007 . The results are shown in Figure 7. The integrated radio halo emission, between $325 \mathrm{MHz}$ and $1.5 \mathrm{GHz}$, has a spectral index of $\alpha_{325}^{1500}=$ $-1.02 \pm 0.04$. This value then steepens between $1.5 \mathrm{GHz}$ and $3.0 \mathrm{GHz}$ to $\alpha_{1500}^{3000}=-1.43 \pm 0.11$. If we instead take the $325 \mathrm{MHz}$ measurement from V13, the halo spectral index is well described by a single power law with index $-1.32 \pm 0.14$. Therefore it remains unclear whether the radio halo spectrum steepens at higher frequencies because of the different P-band flux densities reported by $\mathrm{O} 07$ and V13. 

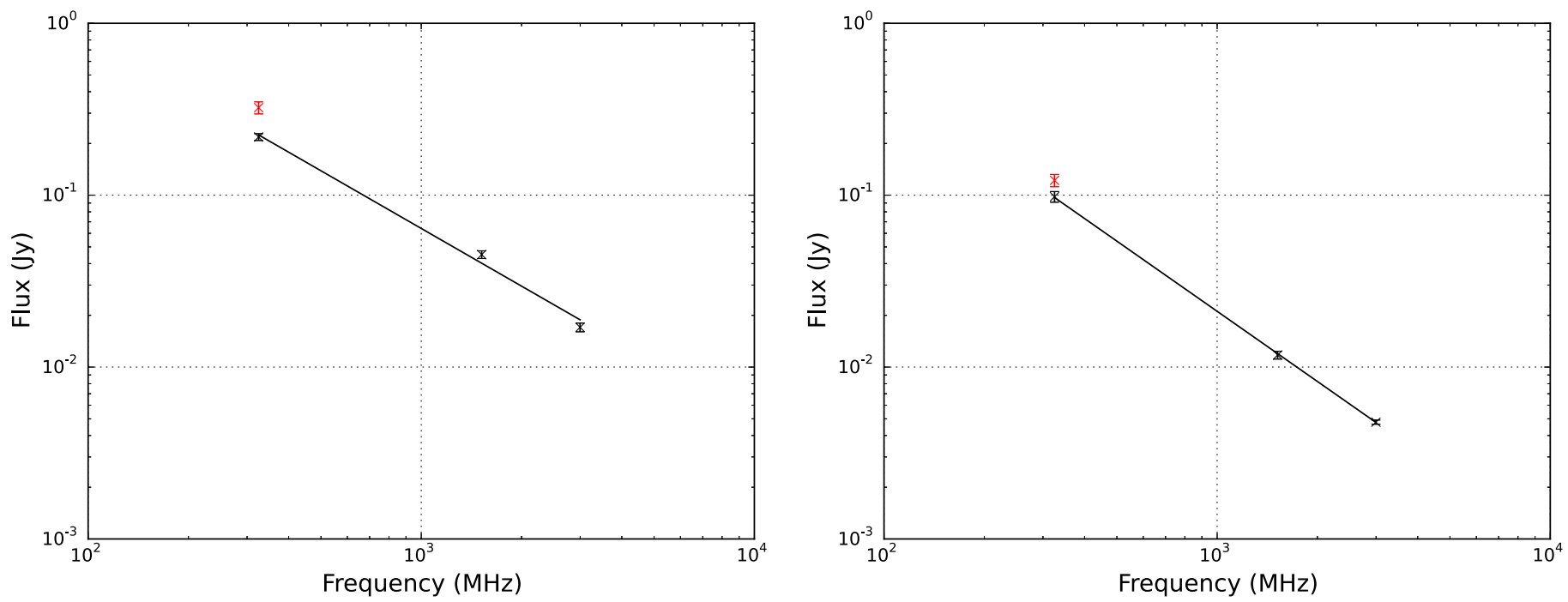

Figure 7. Integrated flux densities of the halo (left) and relic R1 (right) between $325 \mathrm{MHz}$ and $3.0 \mathrm{GHz}$. The 1.5 and $3.0 \mathrm{GHz}$ flux densities are taken from the $30^{\prime \prime}$ radio map. The $325 \mathrm{MHz}$ points are taken from O07 (black) and V13 (red). The black line shows a power-law fit through the black data points.
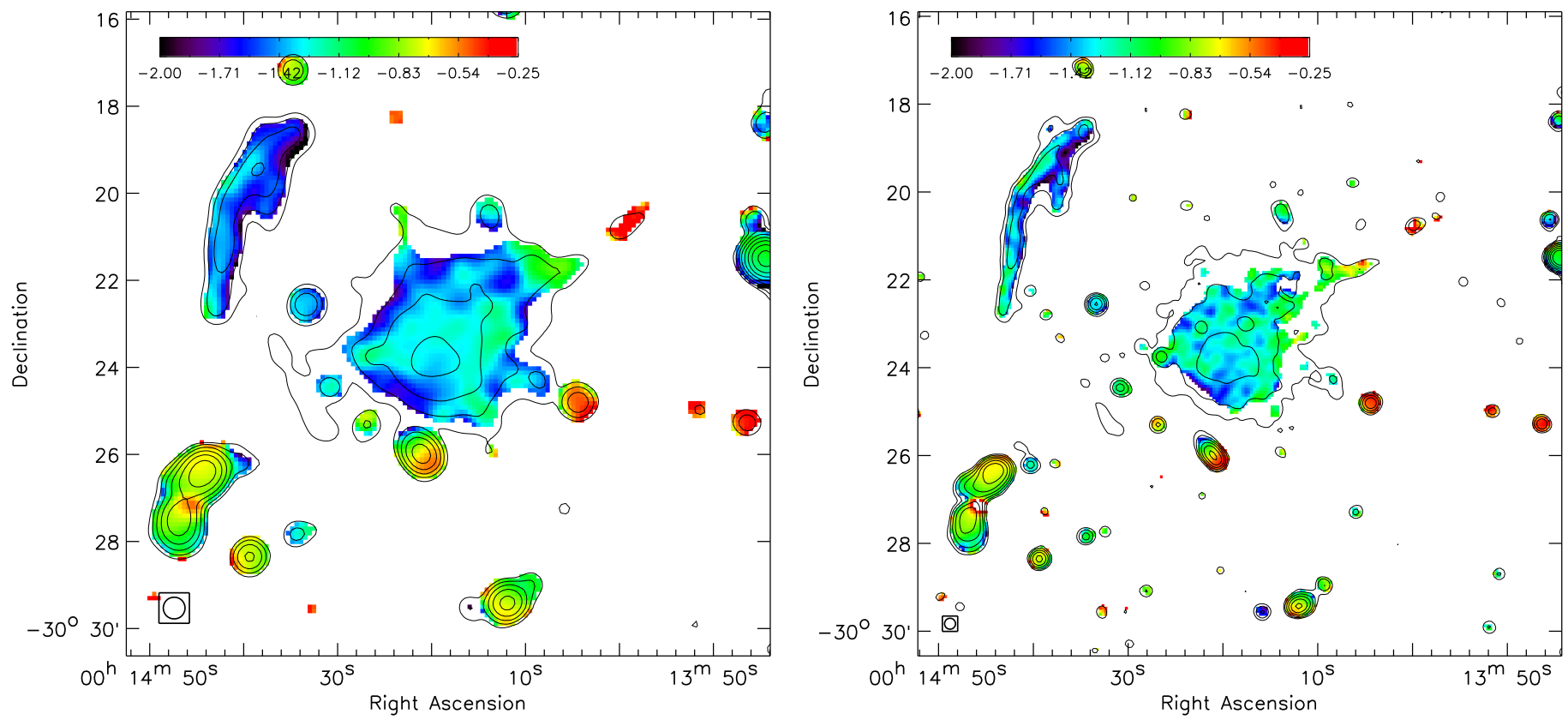

Figure 8. Spectral index map of A2744 between 1.5 and $3.0 \mathrm{GHz}$, tapered to a resolution of $30^{\prime \prime}$ (left) and $15^{\prime \prime}$ (right). Contour levels are obtained from the $1.5 \mathrm{GHz}$ image and placed at levels of $[1,2,4,8, \ldots] \times n \sigma_{\mathrm{rms}}$, with $n=3$ for the left image and $n=4$ for the right image.

Using the flux density from $\mathrm{O} 07$, we find that the integrated radio spectrum from the relic R1 (right panel of Figure 7) over the full range from $325 \mathrm{MHz}$ to $3 \mathrm{GHz}$ is well described by a single power-law spectrum, with a fitted spectral index of $\alpha_{325}^{3000}=-1.36 \pm 0.11$. If we take the flux density from V13 for the power-law fit, we find a consistent result with $\alpha_{325}^{3000}=$ $-1.44 \pm 0.13$.

\subsection{Spectral Index Maps}

To construct spectral index maps we created L- and S-band images with uniform weighting and an inner uv range cut corresponding to the S-band DnC-array data. The $30^{\prime \prime}$ and $15^{\prime \prime}$ resolution maps are shown in Figure 8. The corresponding error maps along with the $10^{\prime \prime}$ and $5^{\prime \prime}$ spectral index maps are displayed in Appendix A in Figures 21 and 22. Only those pixels where the flux values at both frequencies exceed $3 \sigma_{\mathrm{rms}}$ are displayed.
We observe a relatively constant spectral index across the radio halo, with patches of steeper values at its outer boundary. A spectral index gradient across the relic (R1) is evident with values varying from $\sim-0.9$ at the eastern edge to $\sim-2.5$ at the western edge. The gradient is consistently perpendicular to the $\mathrm{N}-\mathrm{S}$ orientation of the relic, with steeper spectral index values in the direction of the cluster center. It is present along the en tire length of the relic, though it exhibits some nonuniformity with the flattest values along the eastern edge ranging from $\sim-0.9$ to $\sim-1.3$. A spectral index gradient oriented toward the cluster center is also present in the emission of diffuse source $\mathrm{R} 4$, with values varying from $\sim-0.8$ to $\sim-1.5$. The elongated emission feature to the NW of the halo (source R3) shows little variation in spectral index along its length (although the spectral index cannot be traced along the full extent of the source) and exhibits flatter spectral values than $\mathrm{R} 1$ at around $\alpha \sim-0.7$. 


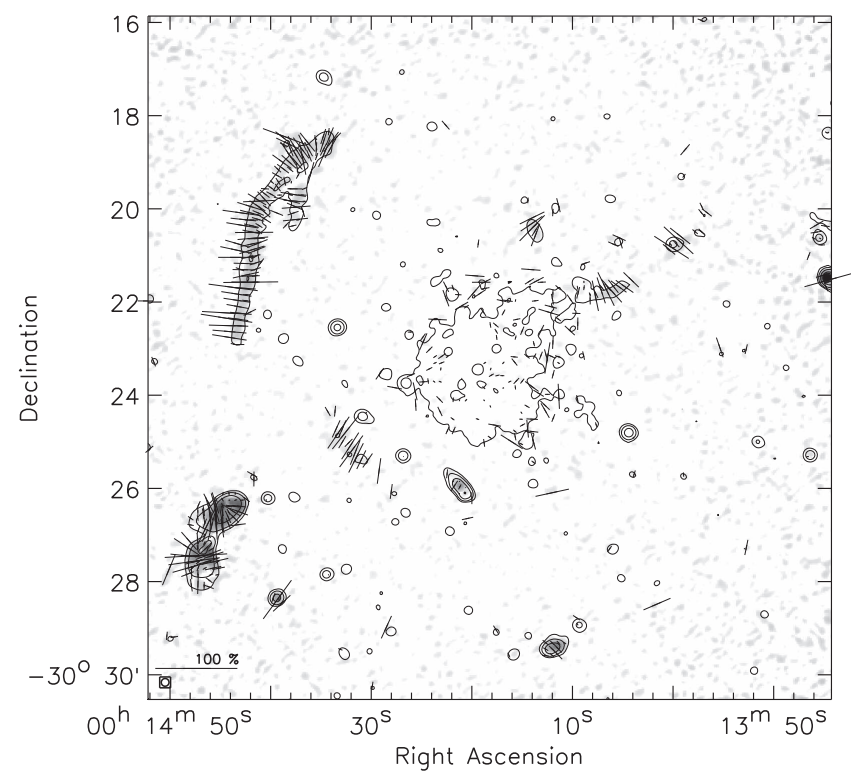

Figure 9. S-band vector map showing the magnitude and orientation of the electric field vectors. Vectors are plotted for every four pixels. The linear polarized intensity image is shown in grayscale. The Stokes $I$ radio contours are from the S-band $10^{\prime \prime}$ uv-tapered image and are plotted at levels of $[1,4,16, \ldots] \times 3 \sigma_{\text {rms }}$.

\subsection{S-band Polarization Map}

S-band images of the Stokes parameters $I, Q$, and $U$ were obtained employing robust $=0$ weighting and a uv taper of $10^{\prime \prime}$. These polarization images serve the purpose of classifying the diffuse extended sources found in the cluster. ${ }^{12}$ These images were corrected for the primary beam attenuation. The polarization angle $(\phi)$ and linear polarized intensity $(p)$ can be determined from the $I, Q$, and $U$ images via

$$
\begin{gathered}
p=\sqrt{Q^{2}+U^{2}} \\
\phi=0.5 \arctan \frac{U}{Q} .
\end{gathered}
$$

A subsequent polarization vector map was constructed from these images, which depicts the magnitude and orientation of the electric field (Figure 9). Polarization vectors are only plotted for pixels above $4 \sigma_{\text {rms }}$ map noise. The polarization vectors shown in Figure 9 are those of the electric field.

It is important to note that we did not correct the polarization vectors for the effect of Faraday rotation. The galactic RM at the location of A2744 is about $6 \mathrm{rad} \mathrm{m}^{-2}$ (Taylor et al. 2009). However, given the relatively high frequency of $2 \mathrm{GHz}$ at the lower end of the S-band and the low galactic RM, corrections for polarization angles would be $<8^{\circ}$. R1, R2, and R4 are located in the cluster outskirts and therefore the contribution from the ICM to the Faraday rotation is likely going to be small as well. For R3, the cluster's RM component might become more important, see Section 5.2.3.

From Figure 9 we find that R1-R4 are highly polarized. The radio halo is mostly unpolarized. The highest polarization fractions $(P)$ are found on the eastern edge of the lower portion of relic R1, with values averaging $\sim 52 \%$. As a whole, R1 has a mean polarization fraction of $27 \%$. The southern portion of the

\footnotetext{
12 A detailed analysis of the Faraday rotation measure (RM), combining all available L- and S-band data, will be presented in a future paper.
}

relic appears to be more strongly polarized than the northern part, with mean values of $33 \%$ and $25 \%$ respectively. We find mean polarization fractions of $30 \%, 43 \%$, and $30 \%$ for sources R4, R2, and $\mathrm{R} 3$ respectively.

The alignment of the $E$-vectors along the length of R1 is consistently perpendicular to the relic's major axis. The gradual change in orientation from south to north suggests that the lower and upper portions of R1 are indeed part of the same physical structure rather than two independent sources seemingly aligned due to projection effects. For component R1-A (Figure 5) the $E$-vectors are aligned in the same direction as the bottom half of the relic. We also find that the $E$-vectors for R2 and R3 are perpendicular to the source elongation, at least in the region where the signal-to-noise ratio is large enough to determine the polarization angles. The interpretation of the polarization map is presented Sections 5.2.1-5.2.4.

\section{Discussion}

\subsection{Radio Halo}

\subsubsection{Spectral Index}

In the turbulent reacceleration model, electrons are reaccelerated via magnetohydrodynamical turbulence (e.g., Schlickeiser et al. 1987; Brunetti et al. 2001; Petrosian 2001). In this case, spectral index variations across radio halos should be related to underlying spatial variations in the turbulent energy and magnetic field strength (Feretti et al. 2004; Orrú et al. 2007; Vacca et al. 2014). The most reliable spectral index map of a radio halo that has been made so far, using LOFAR and VLA data, is the one for the Toothbrush cluster (van Weeren et al. 2016). For this radio halo, the spectral index variations are remarkably small, with an intrinsic scatter of $\leqslant 0.04$, suggesting that the turbulent energy does not change significantly across a region of $0.8 \mathrm{Mpc}^{2}$.

We determined the spectral variations across the radio halo in a similar way to van Weeren et al. (2016). To test the statistical significance of these fluctuations we carried out a study of the distributions of the spectral index value for both the $30^{\prime \prime}$ and $15^{\prime \prime}$ resolution radio images. We extracted flux densities, in both the L- and S-bands, in grids of identically sized boxes placed across the halo (see Appendix A, Figure 23). Spectral index values were then calculated for each individual box.

Only those boxes with a combined L- and S-band flux of

$$
S_{\mathrm{L}}+S_{\mathrm{S}}>5\left(\sigma_{\mathrm{L}}^{2}+\sigma_{\mathrm{S}}^{2}\right)^{1 / 2}
$$

where $\sigma$ is the flux density error, are considered.

The magenta and blue histograms in Figure 10 correspond to the $30^{\prime \prime}$ and $15^{\prime \prime}$ radio images respectively. The distributions have median values of $\left\langle\alpha_{30^{\prime \prime}}\right\rangle=-1.37$ and $\left\langle\alpha_{15^{\prime \prime}}\right\rangle=-1.37$, and standard deviations of $\sigma_{30^{\prime \prime}}=0.18$ and $\sigma_{15^{\prime \prime}}=0.28$.

To test the level of variation in the values we fit a simple zeroth-order polynomial to the data and obtain reduced chisquared values of $\chi_{30^{\prime \prime}}^{2}=1.50$ and $\chi_{15^{\prime \prime}}^{2}=0.68$. The value of 0.68 indicates that the observed fluctuations at $15^{\prime \prime}$ are predominantly the result of measurement errors and fall within the bounds of statistical noise. Interestingly though, the value of 1.50 indicates that at $30^{\prime \prime}$ resolution we do observe some intrinsic complexity in the spectral index distribution. This is confirmed by comparing the median error value of the distributions with the corresponding standard deviations. As described in Vacca et al. (2014), if the variations in spectral index are the result of measurement errors then we expect our 


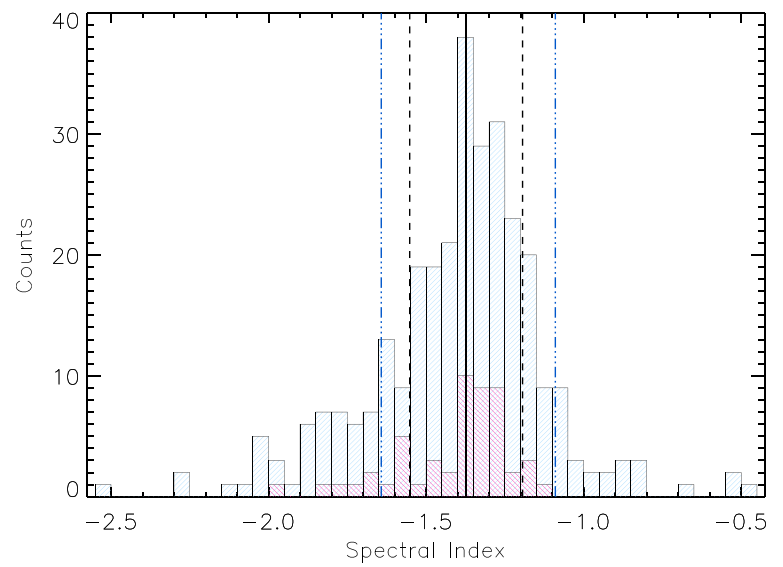

Figure 10. Histogram of spectral index values in the radio halo obtained from the $15^{\prime \prime}$ (in blue) and $30^{\prime \prime}$ (in magenta) resolution radio images. The solid line represents the median value of the $30^{\prime \prime}$ distribution. Black dashed lines mark the $30^{\prime \prime}$ standard deviation about the median value, $\sigma_{30}=0.18$. Blue dashed lines show the $15^{\prime \prime}$ dispersion, $\sigma_{15^{\prime \prime}}=0.28$.

median error value to be of comparable size to the standard deviation. At $15^{\prime \prime}$, since the median error is almost equal in magnitude to the standard deviation, we conclude that the fluctuations in the halo spectral index are not significant. At $30^{\prime \prime}$ resolution, the errors contribute $\sim 50 \%$ to the fluctuations, and they cannot account fully for the level of dispersion. The observed fluctuations at this resolution therefore seem to be statistically significant. This corresponds to variations in the spectral index on spatial scales of $\sim 140 \mathrm{kpc}$. The asymmetric distribution of the histogram (positively skewed) indicates that the observed dispersion at $30^{\prime \prime}$ is predominantly due to the tail of steep spectral index values. From the spectral index map (Figure 8) we can see these values tend to reside at the outer edges of the halo.

We also created a radial profile of the average spectral index value extracted from concentric annuli (see Appendix A, Figure 23), centered on the point of peak surface brightness and spaced with one beam width out to a distance of $150^{\prime \prime}$. The results are shown in Figure 11. We fit a first-order polynomial to the data and find a slope of gradient $(-2.9 \pm 1.7) \times 10^{-4}$ $\mathrm{kpc}^{-1}$. This suggests that there is indeed a mild trend of radial steepening in the spectral index value of the radio halo. We speculate that these steeper regions in the outskirts correspond to regions with less efficient turbulent reacceleration.

In the top right panel of Figure 11 we present the azimuthally averaged radio brightness profiles of the radio halo at 1.5 and 3.0 $\mathrm{GHz}$. Each data point corresponds to the average surface brightness in the same concentric annuli used for the radial spectral index profile.

As suggested by Murgia et al. (2009), we modeled the brightness profile using a simple exponential law of the form

$$
I(r)=I_{0} e^{-\frac{r}{r_{e}}}
$$

where $I_{0}$ is the central radio brightness and $r_{e}$ is the $e$-folding radius. From this we derive values of $I_{0,1.5 \mathrm{GHz}}=3.37 \pm$ $0.04 \mu \mathrm{Jy} \operatorname{arcsec}^{-2}$ and $I_{0,3.0 \mathrm{GHz}}=1.39 \pm 0.05 \mu \mathrm{Jy} \operatorname{arcsec}^{-2}$ for the central radio brightness and $r_{e, 1.5 \mathrm{GHz}}=257.9 \pm 2.4 \mathrm{kpc}$ and $r_{e, 3.0 \mathrm{GHz}}=248.0 \pm 6.1 \mathrm{kpc}$ for the $e$-folding radius. The fact that the $e$-folding radius differs in our observations at the L-band and S-band frequencies is consistent with the previous indication of a slight steepening of the spectral index with radial distance.

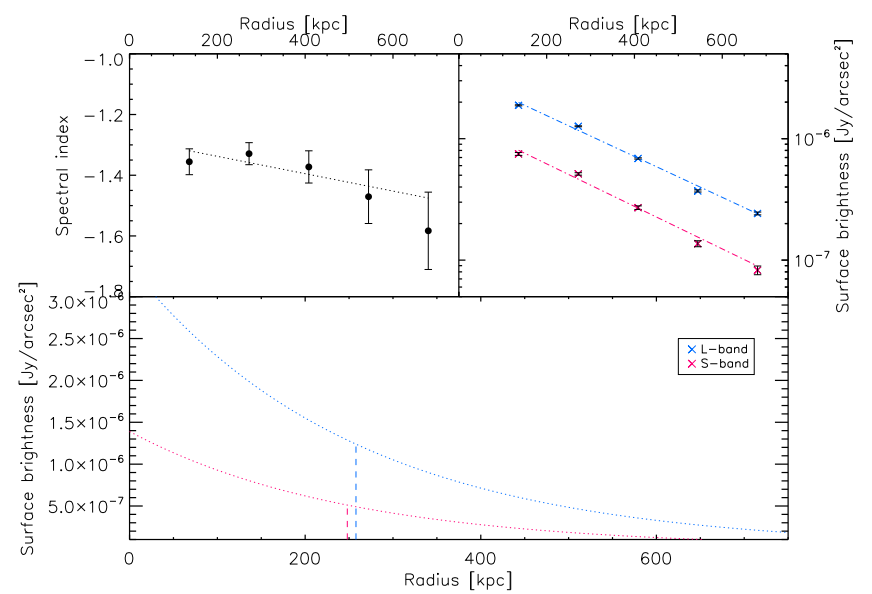

Figure 11. Top left: radial profile of the spectral index from the cluster center. Each value corresponds to the average spectral index in concentric annuli centered on the peak of halo brightness and spaced with one $30^{\prime \prime}$ beam width. The dashed line represents the best fit to the data. Top right: azimuthally averaged surface brightness profiles in the L-band (blue) and S-band (magenta). Values have been extracted from the same concentric annuli as above. Dashed lines represent an exponential fit to the data (Equation (6)). Bottom: plot of the exponential fit to the surface brightness profiles vs. distance to the cluster center for both frequency bands. Vertical lines represent the corresponding $e$-folding radii $\left(r_{e}\right)$.

To further investigate whether the derived spectral indices vary across the radio halo, and are not affected by possible offsets, ${ }^{13}$ we created so called T-T plots (Turtle et al. 1962). These T-T plots compare the flux densities at two frequencies, fitting a straight line through them, and are a useful tool to take into account a nonzero background map level. The resulting reduced $\chi^{2}$ provides a measure to determine whether the spectral index is constant across the source (reduced $\chi^{2} \approx 1$ ) or varies (reduced $\chi^{2}>1$ ). If the reduced $\chi^{2}$ is indeed about one, and there are no issues with a nonzero background, the fit offset should be consistent with being zero (i.e., a straight line through the origin).

For the radio halo, we created a $\mathrm{T}-\mathrm{T}$ plot using the flux densities extracted in $30^{\prime \prime}$ square boxes from the same $\mathrm{S}$ - and L-band image used for the spectral index analysis at $30^{\prime \prime}$ resolution, Figure 25 (left panel). These are the same regions used to create the magenta histogram in Figure 10 . We then fit a straight line through these data points using the MPFITEXY routine (Williams et al. 2010), which utilizes the MPFIT package (Markwardt 2009). For the radio halo we find $\chi^{2}=71.7$ with dof $=50$. This does indeed indicate that the spectral index varies slightly across the radio halo.

\subsubsection{Spatial Correlation Between Radio Spectral Index and X-Ray Temperature}

Orrú et al. (2007) reported the presence of a spatial correlation between radio spectral index and ICM temperature in A2744, with the hotter regions of the ICM corresponding to flatter spectral indices. It was argued that this correlation provided support for the turbulent reacceleration model. We repeated the investigation from Orrú et al. using our new radio data and the deeper Chandra observations.

To create a temperature map, we first divided the cluster into individual regions whose edges follow the X-ray surface brightness using contbin (Sanders 2006). We required a signal-to-noise ratio of at least 55 in the $0.5-7.0 \mathrm{keV}$ band for the

\footnotetext{
${ }^{13}$ For example, from a nonzero background due to missing short spacings.
} 

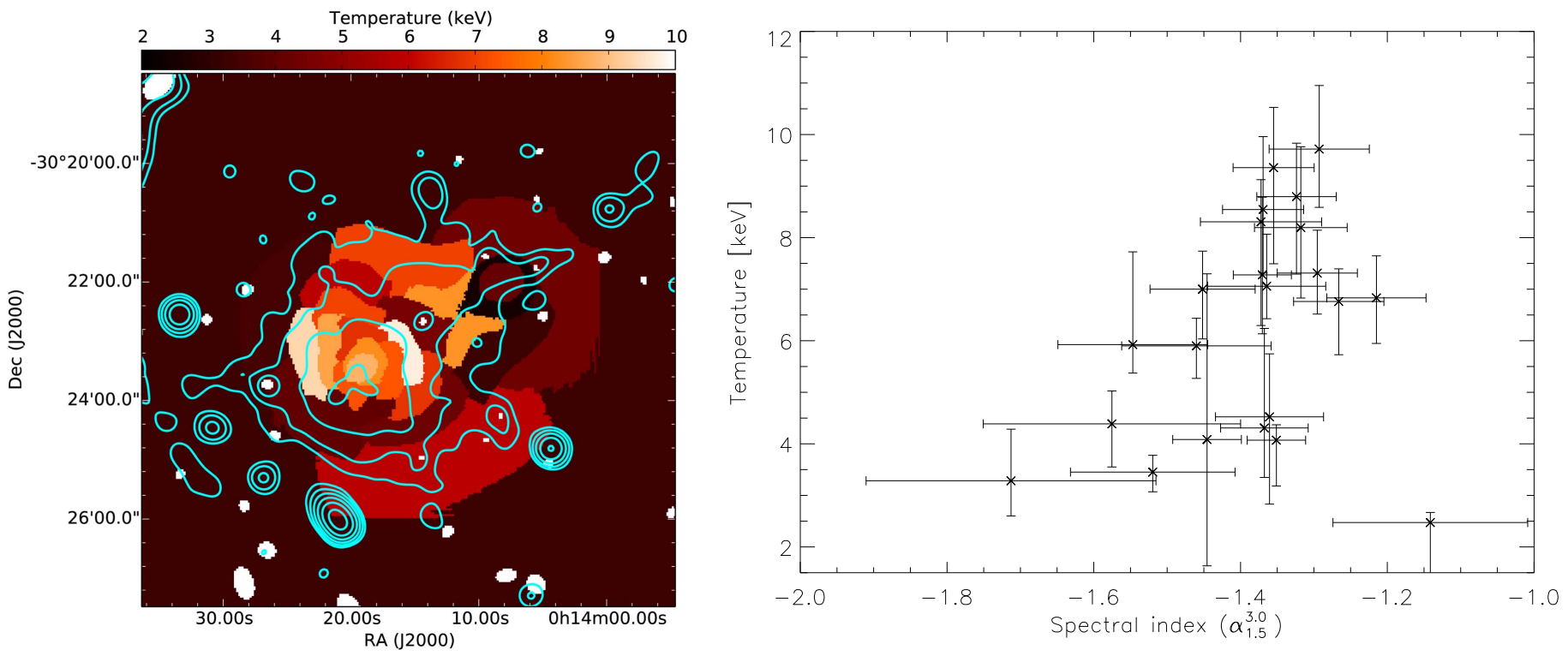

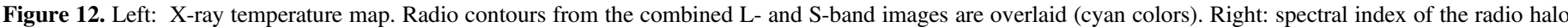

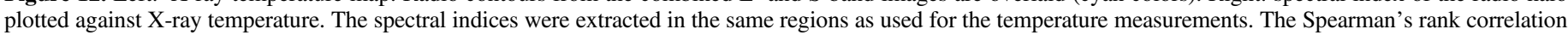
$p$-value is 0.32 , i.e., there is a $32 \%$ probability that the data represent an uncorrelated arrangement of points.

binning. All compact sources were masked. The extracted spectra were fit with XSPEC (v12.8.2, Arnaud 1996). For the fitting we used an absorbed thermal emission model (phabs * APEC). The metallicity was fixed to a value of $0.3 Z_{\odot}$ using the abundance table of Anders \& Grevesse (1989). The redshift was fixed to $z=0.308$. For the Galactic H I column we took a value of $N_{\mathrm{H}}=1.38 \times 10^{20} \mathrm{~cm}^{-2}$ (the weighted average $N_{\mathrm{H}}$ from the Leiden/Argentine/Bonn survey, Kalberla et al. 2005).

The temperature map is shown in Figure 12. We then extracted radio flux densities in the same regions to compute the spectral indices $\left(\alpha_{1.5}^{3.0}\right)$. In Figure 12 we plot the spectral indices against the X-ray temperature. We carried out a Spearman's rank correlation test to search for a possible correlation between spectral index and temperature. From this test we find a $p$-value of 0.32 , i.e., the probability that the data represents an uncorrelated arrangement of points. ${ }^{14}$ Therefore, we conclude there is no strong evidence for the existence of a correlation between radio spectral index and $\mathrm{X}$-ray temperature. Given our better quality data (in both radio and X-rays), this result should be more reliable than Orrú et al. (2007). We note that Orrú et al. (2007) did not provide a statistical measure of the significance of the correlation. Our results are also consistent with recent work by Vacca et al. (2014) and van Weeren et al. (2016). We therefore conclude that currently there is no convincing evidence of the existence of a spatial correlation between X-ray temperature and spectral index for individual radio halos.

\subsubsection{Southeastern Boundary of the Radio Halo}

It is interesting to note that the SE boundary of the radio halo is relatively well defined and aligns with the southern shock reported by Owers et al. (2011). Very similar configurations are observed for the Toothbrush cluster (van Weeren et al. 2016), the Bullet cluster (Shimwell et al. 2014), Abell 520 (Markevitch et al. 2005; Markevitch 2010; Vacca et al. 2014), the Coma Cluster (Planck Collaboration et al. 2013; Uchida et al. 2016), and Abell 754

\footnotetext{
14 If we remove the single low-temperature data point in the bottom right corner of Figure 12 (right panel), corresponding to the region surrounding the NW "interloper," the $p$-value decreases to 0.15 .
}

(Macario et al. 2011). Therefore this appears to be a common phenomenon and it does suggest that some relation exists between cluster shocks and radio halos. This possibly reflects a change in the properties of the ICM's turbulence behind the shock front. However, if this turbulence is generated downstream by the shock front, the timescale for the turbulence to decay to the small scales necessary to reaccelerate particles becomes uncomfortably short (Brunetti \& Lazarian 2007; Brunetti \& Jones 2014).

\subsection{Radio Relics}

The leading theory for relics is that they trace particles (re) accelerated at shocks (e.g., Enßlin et al. 1998; Markevitch et al. 2005; Kang \& Ryu 2011; van Weeren et al. 2017a). The radio spectral index is related to the slope of the underlying electron energy distribution $N(E) d E=\kappa E^{-\delta}$, with $\alpha=\frac{1-\delta}{2}$. In the case of diffusive shock acceleration (DSA; Drury 1983), the shock Mach number $(\mathcal{M})$ is related to the spectral index of radio injection $\left(\alpha_{\text {inj }}\right)$ via

$$
\mathcal{M}=\sqrt{\frac{2 \alpha_{\mathrm{inj}}-3}{2 \alpha_{\mathrm{inj}}+1}} .
$$

The observed spectral gradients across relic's width are thought to be indicative of spectral ageing of electrons in the region downstream of the shock (e.g., van Weeren et al. 2010).

For a stationary shock model (i.e., when the radiative lifetime of the synchrotron-emitting electrons is much shorter than the timescale on which the properties of the shock change) with continuous electron injection, the integrated spectral index $\left(\alpha_{\text {int }}\right)$ over the relic can be related to the injection spectral index via the simple relation

$$
\alpha_{\text {int }}=\alpha_{\text {inj }}-0.5 \text {. }
$$

For a number of relics, with the injection spectral index and integrated spectral index measured independently, Equation (8) seems to be a reasonable approximation (e.g., Giacintucci et al. 2008; Bonafede et al. 2012; Hindson et al. 2014). However, a few exceptions have been reported where the integrated spectral 


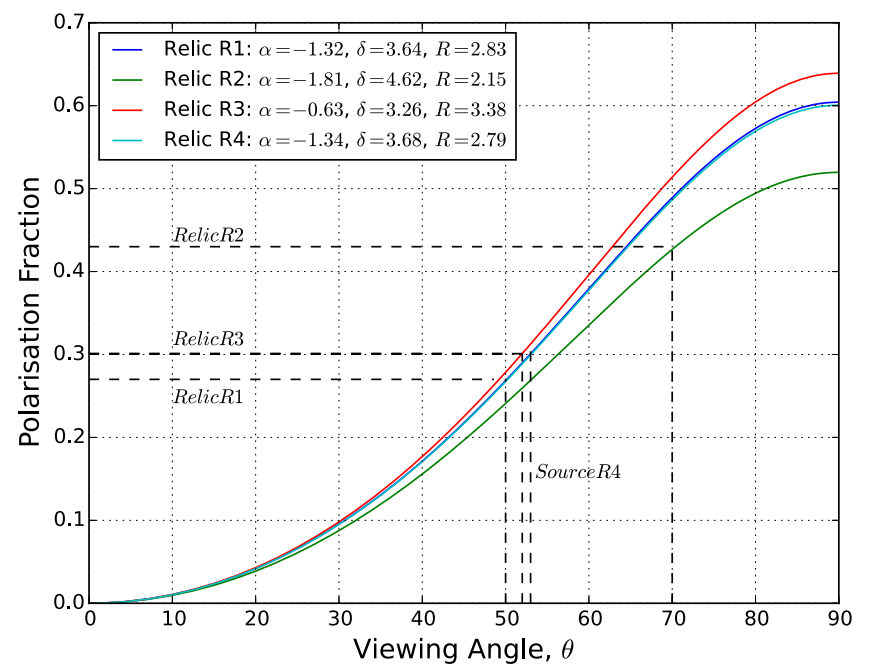

Figure 13. Graph showing the polarization fraction of relics R1, R2, R3, and source R4 plotted as a function of the viewing angle, based on Equation (10). Dashed lines correspond to the observed mean polarization fractions.

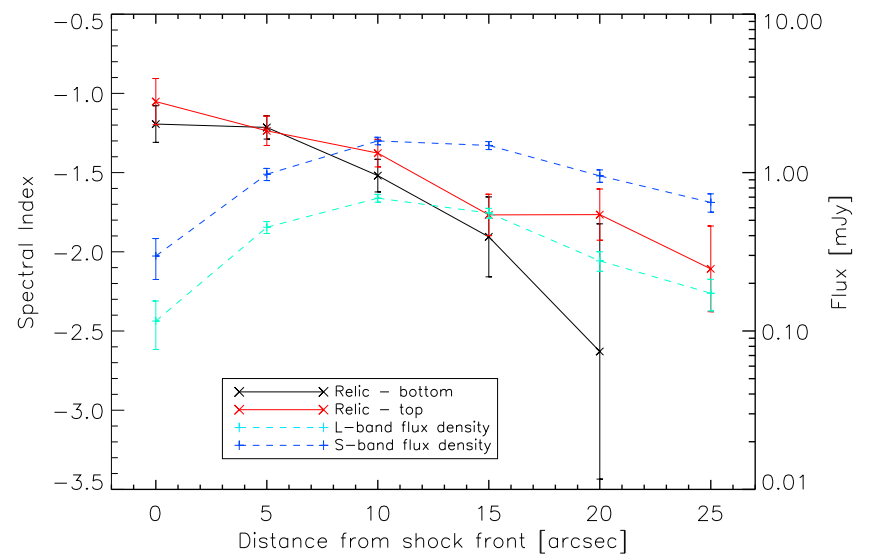

Figure 14. Graph showing the integrated spectral index values from $5^{\prime \prime}$ wide boxes across the relic. The corresponding integrated flux densities used to calculate the spectral indices are shown with dashed lines.

index is rather flat, giving an unphysical result for $\alpha_{\text {inj }}$, i.e., $\alpha_{\text {inj }}>-0.5$ (van Weeren et al. 2012b; Trasatti et al. 2015). Therefore, the usage of Equation (8) should be limited to those cases where $\alpha_{\text {inj }}$ cannot be directly estimated from the data, and any Mach numbers derived from such $\alpha_{\text {inj }}$ values should be treated with caution.

Relics are usually highly polarized. Following Enßlin et al. (1998), the observed polarization fraction of relics can be used to determine a lower limit on the viewing angle of the relic. The alignment of unordered magnetic fields at the shock front should be caused by shock compression, with the degree of alignment dependent on the compression factor. The spectral index $(\alpha)$ of a population of electrons in equilibrium, undergoing acceleration and cooling (i.e., the integrated spectral index), is related to the shock compression ratio, $R$, via (Drury 1983)

$$
R=\frac{\alpha-1}{\alpha+\frac{1}{2}}
$$

where we assume the shocked gas to have a polytropic index of $\gamma=5 / 3$.
The observed polarization fraction also depends on the angle between the line of sight and the $B$-field. The resulting polarization therefore depends on the viewing angle $\theta$ of the shock surface and the compression ratio $R$.

Assuming a weak magnetic field (i.e., the magnetic pressure of the relic is small compared to the internal gas pressure, see Enßlin et al. 1998), the observed polarization fraction of the relic is related to the viewing angle via the formula

$$
\left\langle P_{\text {weak }}\right\rangle=\frac{\delta+1}{\delta+\frac{7}{3}} \frac{\sin ^{2}(\theta)}{\frac{2 R^{2}}{R^{2}-1}-\sin ^{2}(\theta)},
$$

where $\delta$ is the slope of the electron energy distribution (see Section 5.2). Figure 13 plots Equation (10) for all viewing angles and polarization fractions for our sources R1 to R4.

\subsubsection{Relic RI}

In Figure 14 we plot the values of the spectral index, along with their associated flux density measurements, as a function of distance from the shock front. This was done by extracting the flux densities in several $5^{\prime \prime}$ wide box regions. ${ }^{15}$ Given the distinct "wedge-like" shape of the shock front, we perform separate measurements across the top and bottom portions of the relic R1, see Figure 24.

Figure 14 displays a clear spectral gradient for both portions of the relic, with the spectral index values steepening away from the relic's outer edge down to values of $\sim-2.5$. From these plots we obtain a flattest spectral index of $\alpha=-1.12 \pm 0.19$ on the eastern side of the relic, averaging the top and bottom profiles. We also created a T-T plot using the flux densities extracted from the $5^{\prime \prime}$ resolution L- and S-band maps in the region shown in Figure 24. The T-T plot is shown in Figure 25 (right panel). Performing the same procedure as outlined in Section 5.1.1, we find $\chi^{2}=37.3$ with dof $=11$, clearly confirming that the spectral index varies across the relic. ${ }^{16}$

The value $\alpha_{\text {inj }}=-1.12 \pm 0.19$ seems somewhat low compared to $\alpha_{\text {int }}=-1.32 \pm 0.09$, considering Equation (8). However, we note that this $\alpha_{\text {inj }}$ value is measured from a map with finite spatial resolution and that projection effects are not included. Both effects lead to an underestimation of $\alpha_{\text {inj }}$ (van Weeren et al. 2012a). Combining this with the statistical uncertainties of both measurements, the current data do not allow us to draw a firm conclusion on the validity of the assumption of stationary shock conditions for R1.

Using $\alpha_{\mathrm{inj}}=-1.12 \pm 0.19$, we derive a shock Mach number of $\mathcal{M}=2.05_{-0.19}^{+0.31}$ for relic R1. Such a Mach number is consistent with weak merger shocks. Recently, Eckert et al. (2016) reported evidence of a shock at the relic's eastern edge using XMM-Newton and Suzaku X-ray data. Using the Rankine-Hugoniot jump conditions, they derived a shock Mach number of $\mathcal{M}=1.7_{-0.3}^{+0.5}$. Our value is in agreement with this within errors.

Eckert et al. (2016) also derived an estimate for the acceleration efficiency required of electrons at the shock corresponding to $\mathrm{R} 1$ in order to reproduce the observed radio power of the relic. They find that the efficiency required for the

\footnotetext{
$155^{\prime \prime}$ matches the beam size of the spectral index map with the highest resolution.

16 The T-T plot does show a relatively large offset. However, an offset might be expected because of the large reduced $\chi^{2}$, indicating that the data are not well described by a straight line. Therefore, the offset cannot be directly interpreted as a nonzero background level.
} 
R1

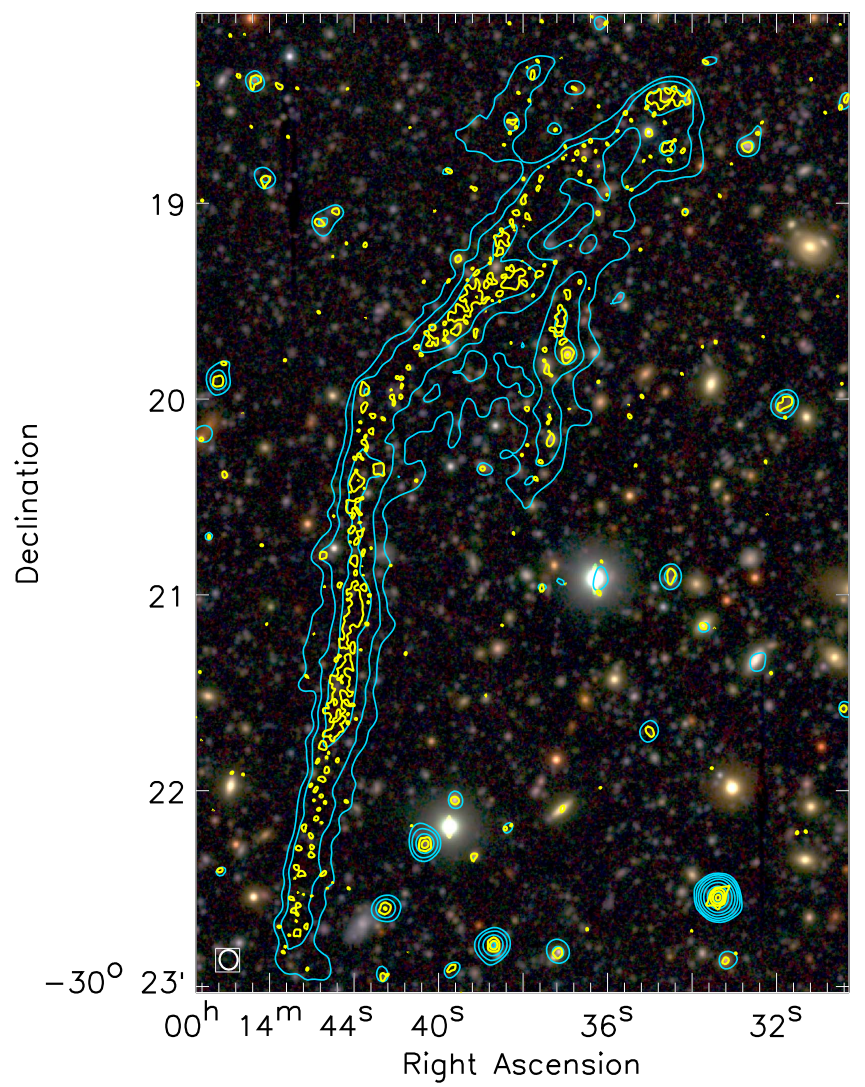

Figure 15. Subaru optical $B R z$ color image (Medezinski et al. 2016) around relic $\mathrm{R} 1$ with radio contours overlaid. The blue and red radio contours are from the $5^{\prime \prime}$ uv-tapered image and the $1-4 \mathrm{GHz}$ wideband image with robust $=0$, respectively. Contour levels are drawn at $[1,2,4, \ldots] \times 3 \sigma_{\text {rms }}$ (blue) and $[1,4,8, \ldots] \times 4 \sigma_{\text {rms }}($ red $)$.

shock is $10-10^{3}$ times higher than that predicted by DSA for thermal electrons. It was suggested that DSA reacceleration of a pool of mildly relativistic fossil electrons, already present in the cluster volume, can reconcile such discrepancies. Given that a variety of sources in the ICM (such as supernovae, AGNs, etc.) are able to supply relativistic electrons with a broad range of energies, this scenario could be viable in the context of weak shock acceleration. Indeed, observational evidence does exist for a connection between tailed radio galaxies and relics (Giovannini et al. 1991; Bonafede et al. 2014; Shimwell et al. 2015; Botteon et al. 2016; van Weeren et al. 2017a, 2017b).

The morphology of R1 with its peculiar extension R1-A could have resulted from the reacceleration of a remnant patch of fossil AGN plasma. In A2744, we do observe a number of compact radio sources embedded within the $\mathrm{R} 1$ relic region, although at present we cannot establish a direct link between any of these sources and the relic, see Figure 15. One compact radio source is embedded within R1-A (R.A. $=3^{\circ} .6538408$, decl. $=-30^{\circ} .3296082$ ), but this seems to be a background source with $z_{\text {phot }}=0.59 \pm 0.07$ (Medezinski et al. 2016).

The observed mean polarization fraction of $27 \%$ of $\mathrm{R} 1$ is typical for radio relics and suggests a high degree of ordering of the magnetic fields $(B)$, aligning them within the shock plane (e.g., Clarke \& Enßlin 2006; Bonafede et al. 2009). It is worth noting that we observe large local fluctuations in this value, particularly toward the southern part of relic R1, where values reach up to a polarization fraction of $\sim 60 \%-70 \%$, which is close to the maximum value for synchrotron radiation (e.g., Rybicki \& Lightman 1986). Using the mean polarization fraction, we determine the viewing angle of relic R1 to be $\theta \gtrsim 50^{\circ}$ (Figure 13). This is only a lower limit, with the actual value likely to be higher due to depolarization. With a viewing angle of $\theta \gtrsim 50^{\circ}$ we can constrain the geometry of the primary NE-SW merger axis to be within $\sim \pm 40^{\circ}$ of the plane of the sky.

\subsubsection{Diffuse Source R2}

R2 has not been identified as an individual source in any observations to date. However, upon closer inspection of the radio maps presented by V13, hints of the sources are visible. Part of this emission was classified as a radio bridge by V13. Our deeper images suggest that $\mathrm{R} 2$ is not a radio bridge but a separate elongated diffuse source with a length of $1.15 \mathrm{Mpc}$ and located $\sim 0.9 \mathrm{Mpc}$ southeast from the cluster core.

The relatively low flux density, particularly in the S-band, prevents the source from showing up in the spectral index maps. However, using the integrated flux densities we obtain an integrated spectral index value of $\alpha=-1.81 \pm 0.26$. We estimate the radio power of the source to be $P_{1.4 \mathrm{GHz}}=$ $(9.59 \pm 0.73) \times 10^{23} \mathrm{~W} \mathrm{~Hz}^{-1}$.

Most importantly, in the $E$-vector polarization map (Figure 9) we find the source to be highly polarized, with a mean value of $43 \%$. Given the peripheral location of the source, its megaparsec size, steep spectrum, and high polarization fraction, we conclude that this source is a new radio relic. Henceforth we will refer to source R2 as relic R2. The polarization vector map shows the $E$-vectors to be aligned perpendicular to the SE edge of the relic. Based on this and the relic's location and orientation, we suggest that the relic is the product of a shock front traveling in the SE direction. Using Equation (10), we derive a viewing angle of $\theta \gtrsim 70^{\circ}$ (see Figure 13), meaning we are seeing the relic close to edge-on.

Due to the relatively low flux density of R2, it is difficult to identify any specific region of the relic associated with the point of particle injection at the shock front. However, if we make the assumption that the R2 shock follows a simple continuous injection model with $\alpha_{\text {int }}=\alpha_{\text {inj }}-0.5$, we obtain an estimate for the injection spectral index of $\alpha_{\mathrm{inj}}=-1.31 \pm 0.26$. This corresponds to a shock with Mach number $\mathcal{M}=1.86_{-0.17}^{+0.29}$.

To search for the presence of a shock at the location of R2, we extracted a surface brightness profile in the $0.5-2.0 \mathrm{keV}$ band with PyXel (Ogrean 2016, 2017) in a sector centered on R. A. $=3.553916$, decl. $=-30^{\circ} .36725$. We used opening angles between $190^{\circ}$ and $230^{\circ}$. This sector was chosen to match the radius of curvature of the relic R2, see Figure 6. Regions with compact X-ray sources were excluded. The instrumental and sky backgrounds were subtracted.

We fitted a broken power-law density model to the surface brightness (see Equation (11)) with $\mathrm{PyXel}$, assuming that the emissivity is proportional to the density squared:

$$
n(r)= \begin{cases}\left(n_{2} / n_{1}\right) n_{0}\left(\frac{r}{r_{\text {edge }}}\right)^{a_{2}}, & r \leqslant r_{\text {edge }} \\ n_{0}\left(\frac{r}{r_{\text {edge }}}\right)^{a_{1}}, & r>r_{\text {edge }} .\end{cases}
$$

In the above equation the subscripts 1 and 2 denote the upstream and downstream regions, respectively. The parameter $n_{2} / n_{1} \equiv R$ is the electron density jump (i.e., compression 


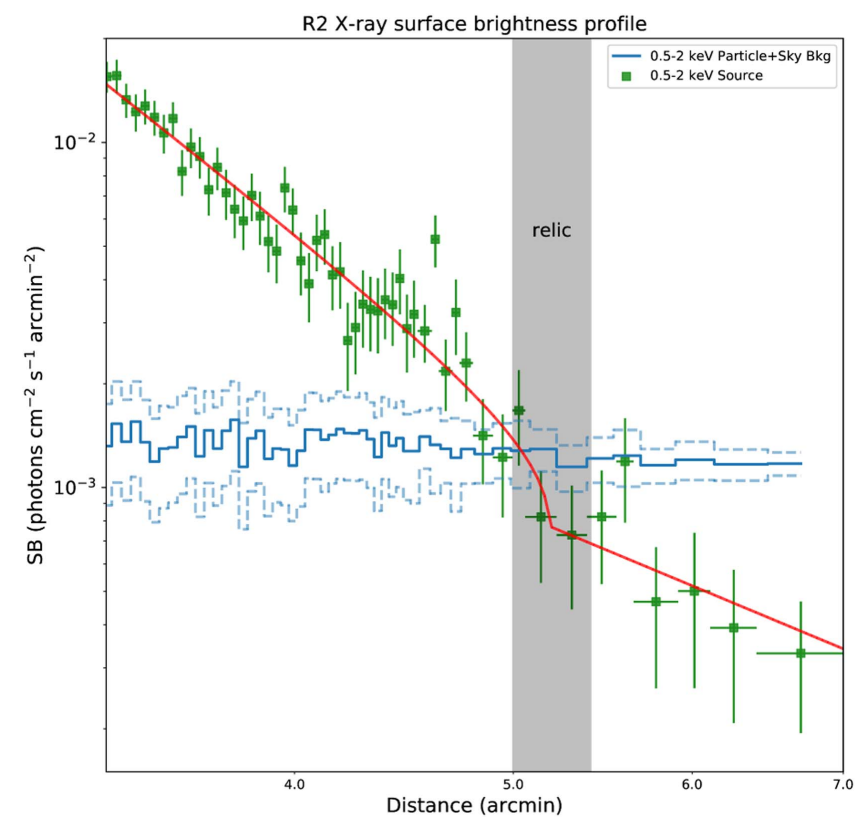

Figure 16. X-ray surface brightness profile across relic R2 in the sector shown in Figure 6, with the distance increasing outward to the SE. The red line shows the best-fitting broken power-law density model (Equation (11)). The relic's location is indicated by the gray-shaded region. The blue solid and dashed lines show the total (sky and particle) background and corresponding uncertainty, respectively.

ratio). The parameters $a_{1}$ and $a_{2}$ define the slopes of the power laws, $n_{0}$ is a normalization factor, and $r_{\text {edge }}$ is the position of the jump. The model is then integrated along the line of sight assuming spherical symmetry. The uncertainties on the bestfitting parameters are determined using a Markov chain Monte Carlo (MCMC) method (Foreman-Mackey et al. 2013).

For a shock, $n_{2} / n_{1}$ is related to the Mach number $(\mathcal{M})$ via the Rankine-Hugoniot condition (Landau \& Lifshitz 1959)

$$
\mathcal{M}=\left[\frac{2 R}{\gamma+1-R(\gamma-1)}\right]^{1 / 2},
$$

where we take $5 / 3$ for the adiabatic index of the gas $(\gamma)$.

From the surface brightness profile we see some evidence for a change in the slope at a radius of about $5^{\prime}$ (see Figure 16). The best-fitting double power-law model finds the presence of a density jump with $R=1.39_{-0.22}^{+0.34}$ at $r_{\text {edge }}=5.15_{-0.13}^{\prime+0.16}$. All uncertainties are quoted at the $1 \sigma$ level. The corresponding MCMC "corner plot" for the distribution of the uncertainties in the fitted parameters is shown in Figure 26, Appendix B. Although the jump is significant given the $1 \sigma$ uncertainties, we cannot completely rule out the possibility of a statistical fluctuation (about a 10\% chance). If we assume that this possible density jump traces a shock it would correspond to a shock Mach number of $\mathcal{M}=1.26_{-0.15}^{+0.25}$, with the shock traveling to the SE. If the relic traces an outward traveling shock it must be located on the SE side of the relic. The best fit location of the density jump is slightly closer to the NW side of the relic. However, the relic's outer SE edge is still located within the $90 \%$ confidence limits of $r_{\text {edge }}$.

We conclude that R2 likely traces a shock given (1) that it probably traces a density jump, (2) that the source is located in the cluster outskirts, and (3) the observed radio properties (megaparsec extent and polarization properties). We note that the surface brightness edge we find is not the same one as reported by Owers et al. (2011). The orientation of the shock reported by Owers et al. and the one corresponding to R2 are roughly parallel; however, they are not directly related given that the separation between the two is $\sim 0.5 \mathrm{Mpc}$. Interestingly, the edge is located in a sector just above (further clockwise than) the one taken by Markevitch (2010), where a very low-contrast jump was reported. Since the edge is located at approximately the same clustercentric distance as the edge seen by Markevitch (2010), it is likely an extension of same shock front. Therefore, we conclude that there are two shocks fronts in the SE of the cluster with similar orientations, but separated by $\sim 0.5 \mathrm{Mpc}$. The presence of a bullet-like feature detected by Owers et al. (2011) south of the main core (Figure 6) does suggest a merger event of the main core with a smaller substructure seen just after core passage. This merger event likely created the shock front reported by Owers et al. (2011). Given its location, the shock corresponding to $\mathrm{R} 2$ must have formed from an earlier merger event. The orientation of the R2 shock favors a NW-SE merger axis, along the axis where NW and S3 and S4 components are located (Figure 1). However, given the complexity of the A2744 cluster with multiple ongoing mergers, it is hard to draw firm conclusions and identify which of these components could have been involved in the merger event that created the R2 shock.

For the outer edge corresponding to $\mathrm{R} 2$, the X-ray-derived Mach number $\left(\mathcal{M}=1.26_{-0.15}^{+0.25}\right)$ is lower than the radio-derived Mach number $\left(\mathcal{M}=1.86_{-0.17}^{+0.29}\right)$. Similar discrepancies have been seen before (e.g., van Weeren et al. 2016), and this was invoked as evidence against DSA, although the radio-derived Mach number was based on the measured integrated spectral index and not on a more reliable measured injection spectral index (see Kang 2015). Furthermore, the discrepancy is not very large given the statistical uncertainties and the simplifying assumptions made in fitting the X-ray surface brightness profile. As such, it is hard to comment on the likely acceleration model for R2 based on the difference between the Mach numbers derived from the radio spectral index and from X-rays. On the other hand, shocks with $\mathcal{M}<2$ should be very inefficient accelerators and therefore a reacceleration scenario might be preferred. Although several compact, faint radio sources are located in the relic's vicinity, we do not identify a clear candidate for supplying fossil radio plasma for a reacceleration scenario, see Figure 17.

\subsubsection{Diffuse Source R3}

Similarly to R2, parts of source R3 were detected in the radio maps produced by V13, though it was not identified as an individual source. In those observations it was deemed to be an elongated extension of the halo and was dubbed the "northwestern ridge."

At high resolution $\left(\gtrsim 15^{\prime \prime}\right)$ the emission is seen as a long $(\gtrsim 1.1 \mathrm{Mpc}$ ) filament protruding from the NW edge of the halo, out to a distance of $\sim 1.6 \mathrm{Mpc}$. The emission appears to arc somewhat to the north as it extends away from the cluster center. Projection effects make it difficult to determine whether the structure is connected to the halo or whether it is an independent feature. As such, our estimate of a physical size should be considered a lower limit.

We estimate the monochromatic radio power of the source to be $P_{1.4 \mathrm{GHz}}=(4.72 \pm 0.45) \times 10^{23} \mathrm{~W} \mathrm{~Hz}^{-1}$. However, this is potentially an overestimate. The relic region was drawn using the polarization intensity map as a guide. As shown in Figure 5, the resulting region extends partway into the halo. The subsequent flux density measurements are likely contaminated with halo flux.

The source is also highly polarized along its length with a mean polarization fraction of $\sim 30 \%$. Based on the filament-like morphology, large size, and high polarization fraction we also 


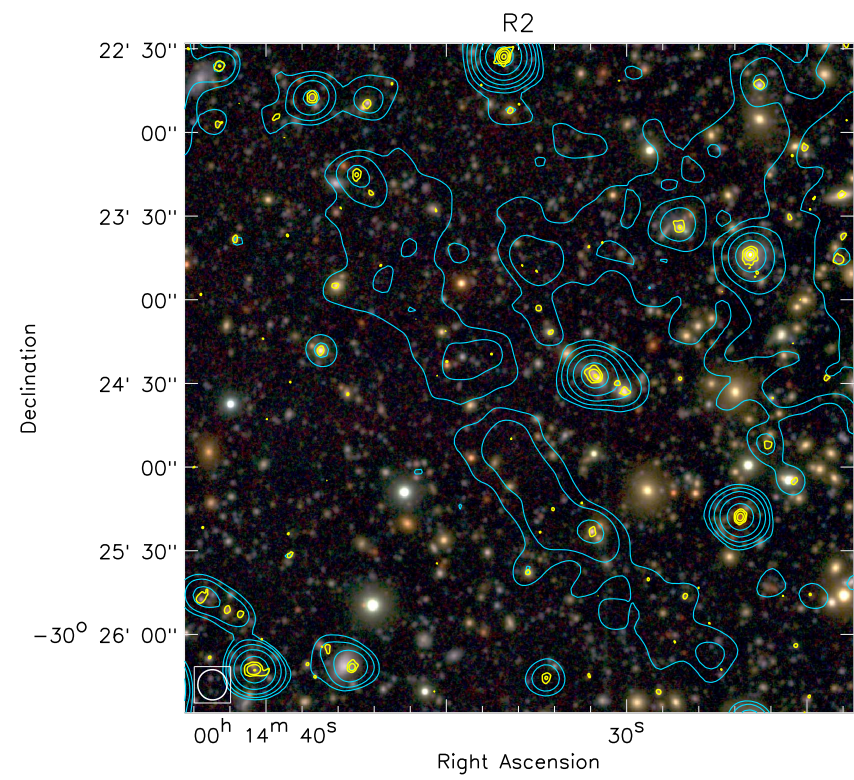

Figure 17. Subaru optical $B R z$ color image (Medezinski et al. 2016) around relic $\mathrm{R} 2$ with radio contours overlaid. The blue and red radio contours are from the $10^{\prime \prime}$ uv-tapered image and the $1-4 \mathrm{GHz}$ wideband image with robust $=0$, respectively. Contour levels are drawn at $[1,2,4, \ldots] \times 3 \sigma_{\text {rms }}$ (blue) and $[1,4,8, \ldots] \times 4 \sigma_{\text {rms }}($ red $)$

classify this source as a newly discovered radio relic. Henceforth we shall refer to source R3 as relic R3. Interestingly, the orientation of the aligned $E$-vectors perpendicular to the relic's orientation (Figure 9) suggests a shock propagation direction indicative of an off-center $\mathrm{N}-\mathrm{S}$ minor merger. A caveat is that R3 is partly projected on top of the NW "interloper." Therefore, the polarization vectors might have been affected by Faraday rotation, depending on the (unknown) relative location of the radio emission with respect to the magnetized ICM.

The relic possesses one of the flattest spectra observed in radio relics with an integrated spectral index value of -0.63 . Such a flat spectrum is quite rare and one cannot assume a simple stationary shock model to explain the spectrum. The reason is that in the limit of strong shocks $(\mathcal{M} \gg 1)$ the particle energy distribution, $\delta$, approaches an asymptotic flat value of -2 . This means that the injection spectral index cannot be flatter than -0.5 , whereas we would obtain a value of -0.13 if we were to add 0.5 to the value of -0.63 (Equation (8)). If the measured integrated spectral index value is the injection spectrum, we would also be observing a rather strong shock. Based on an injection value of $\alpha_{\text {inj }}=-0.63 \pm 0.21$ the shock would have a Mach number measuring $\mathcal{M}=4.04 \pm 1.4$.

It is possible we are observing the integrated spectrum well before the break frequency, whilst the emission is determined


relic is "young," and stationary shock conditions do not (yet) apply (e.g., van Weeren et al. 2012b; Trasatti et al. 2015). Alternatively, the flat spectrum reflects the slope of the fossil distribution in the case of reacceleration, or a combination of the two above (reacceleration + nonstationary shock conditions, Kang \& Ryu 2015; Kang 2015). A radio-optical overlay does not reveal any obvious source of fossil radio plasma, Figure 18. The relatively bright compact source at $\mathrm{R}$. A. $=3^{\circ} .4999688$, decl. $=-30^{\circ} .3459524$ has $z_{\text {phot }}=0.46 \pm$ 0.05 (Medezinski et al. 2016) and is thus likely a background object and not associated with R3.

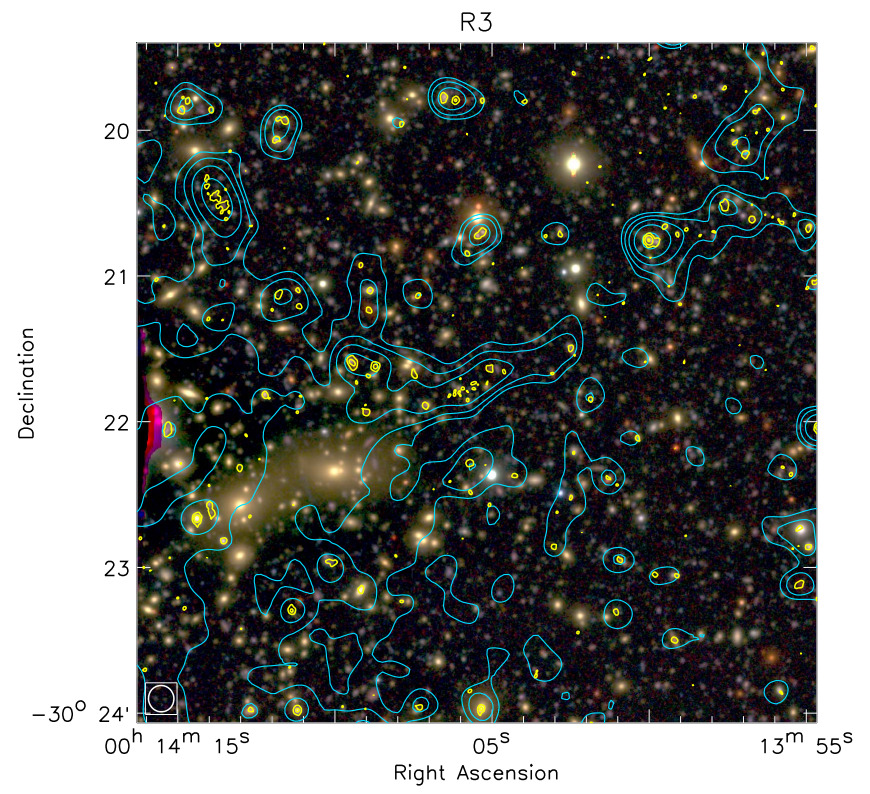

Figure 18. Subaru optical $B R z$ color image (Medezinski et al. 2016) around relic R3 with radio contours overlaid. The blue and red radio contours are from the $10^{\prime \prime}$ uv-tapered image and the $1-4 \mathrm{GHz}$ wideband image with robust $=0$, respectively. Contour levels are drawn at $[1,2,4, \ldots] \times 3 \sigma_{\text {rms }}$ (blue) and $[1,4,8, \ldots] \times 4 \sigma_{\text {rms }}($ red $)$.

Using the mean polarization fraction, we derive a viewing angle of $\theta \gtrsim 52^{\circ}$ for R3, see Figure 13. Purely from a visual inspection, one would probably expect the relic to be viewed close to perfectly edge-on, considering that it measures just $150 \mathrm{kpc}$ across at its widest point. Interestingly, the relic appears to sit exactly on top of the NW "interloper" gas clump/ core, whereas shock fronts should typically lead gas cores. We see no gas cores on either side of the relic. Therefore, the positioning of the radio shock front/relic perhaps implies that the shock plane is located in front of or behind the NW "interloper" and just projected on top of it.

\subsubsection{Diffuse Source R4}

Diffuse source R4 consists of a small patch of diffuse emission (Figures 1, 4, and 20). Like R2 and R3, it too appears in the observations of $\mathrm{V} 13$, and even in the $325 \mathrm{MHz}$ radio map of O07, though it is partly blended with the halo.

$\mathrm{R} 4$ measures just $50 \times 30 \mathrm{kpc}^{2}$ in size and is located $\sim 1 \mathrm{Mpc}$ north-northwest from the cluster core. We find no obvious optical counterpart in the vicinity, see Figure 20. Most intriguingly, this small patch appears to be highly polarized at $\sim 30 \%$, with $E$-vectors aligned perpendicular to the source elongation, just like a typical radio relic. We estimate a monochromatic radio power of $P_{1.4 \mathrm{GHz}}=(3.0 \pm 0.3) \times 10^{23} \mathrm{~W} \mathrm{~Hz}^{-1}$ for the source. It is the only diffuse source other than R1 to exhibit a spectral index gradient, with $\alpha$ steepening from $\sim-0.8$ to $\sim-1.5$ in the $\mathrm{N}-\mathrm{S}$ direction, with an average integrated spectral index of $\alpha=-1.34 \pm 0.24$. This integrated spectral index is consistent with the flattest spectral index we observe under the assumption of stationary conditions, i.e., Equation (8). Using the integrated spectral index and Equations (7) and (8), we calculate a shock Mach number of $\mathcal{M}=2.62_{-0.50}^{+1.76}$.

In the introduction, we mentioned three different types of radio relics: giant radio relics, AGN relics, and radio phoenices. These can also be divided more generally, as in Feretti et al. (2012), into "elongated" and "roundish" relics. Elongated relics in this case 


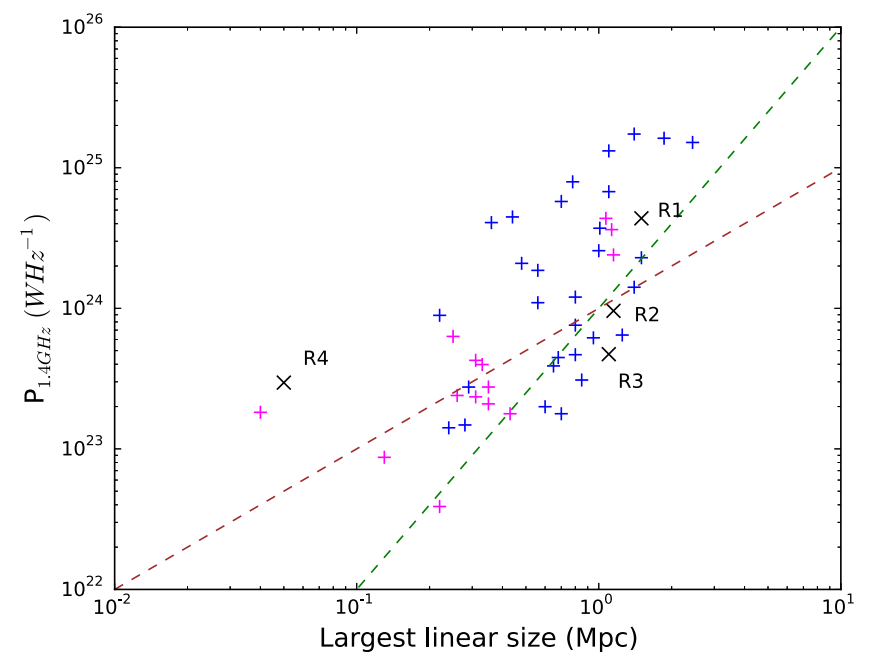

Figure 19. 1.4 GHz monochromatic radio power vs. largest linear size (LLS) for a sample of known radio relics taken from Feretti et al. (2012). "Elongated" relics are shown in blue. "Roundish" relics are shown in magenta. In black, and labelled with $\times$, are the four candidate radio relics discussed in this paper. The two dashed lines represent the cases where $P_{1.4 \mathrm{GHz}} \propto$ LLS (red) and $P_{1.4 \mathrm{GHz}} \propto \mathrm{LLS}^{2}$ (green).

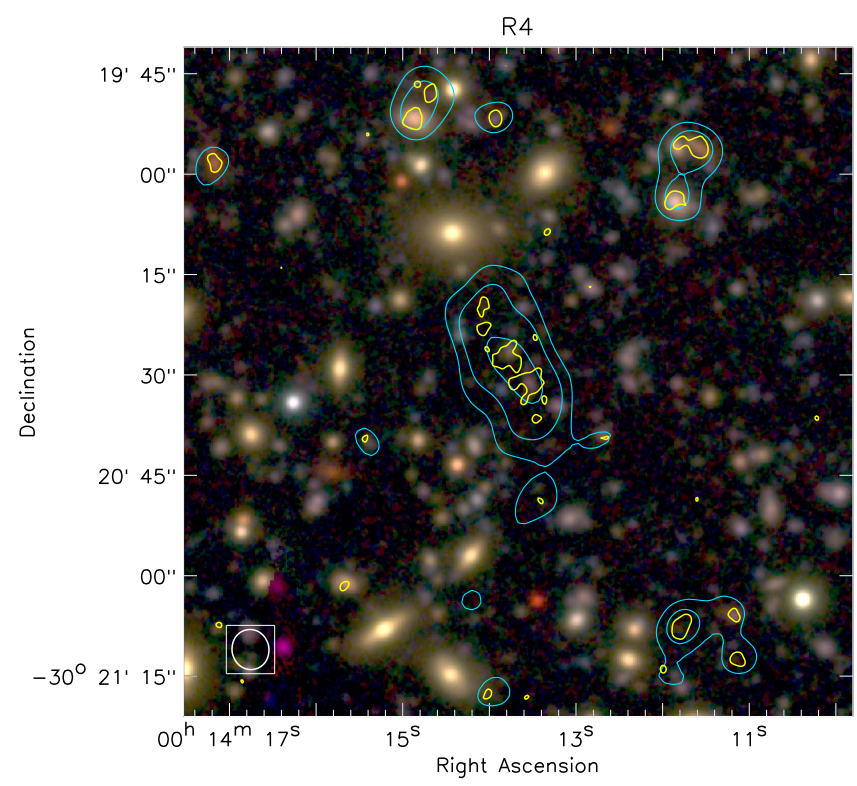

Figure 20. Subaru optical $B R z$ color image (Medezinski et al. 2016) around relic $\mathrm{R} 4$ with radio contours overlaid. The blue and red radio contours are from the $5^{\prime \prime}$ uv-tapered image and the $1-4 \mathrm{GHz}$ wideband image with robust $=0$, respectively. Contour levels are drawn at $[1,2,4, \ldots] \times 3 \sigma_{\text {rms }}$ (blue) and $[1,4,8, \ldots] \times 4 \sigma_{\text {rms }}($ red $)$

simply correspond to the standard giant radio relics we previously defined. "Roundish" relics consist of diffuse extended radio sources with more regular and roundish morphology, although some of them display complex filamentary shapes. They too tend to be located at smaller distances from the cluster center. The key defining feature though is that of a steep and curved integrated radio spectrum. It is thought that they consist of AGN relics and radio phoenices, though other explanations have also been suggested, such as buoyant radio bubbles.

A relation has been shown to exist between the radio power of a relic and its LLS, with larger relics possessing more power. For large relics seen edge-on one would expect that the depth of a relic scales roughly with the LLS, and thus $P_{1.4 \mathrm{GHz}} \propto \mathrm{LLS}^{2}$. However, the correlation becomes more complicated when "elongated" or "roundish" relics are included. In Figure 19 we overlay the results for our four relic candidates onto a plot of the radio power at $1.4 \mathrm{GHz}$ against the LLS of a sample of relics taken from Feretti et al. (2012). Shown in blue are relics classified as elongated; roundish relics are shown in magenta.

In general, elongated relics have greater radio power than roundish ones, with the exception of A1664, A2256, and A2345W, as previously noted by Feretti et al. (2012). Both types can have low radio powers, $\sim 1 \times 10^{23} \mathrm{~W} \mathrm{~Hz}^{-1}$, but of these, elongated relics tend to have a larger size. Relics R1, R2, and R3 fall nicely into the main group of elongated relics. Source R4, on the other hand, appears to fall into the category of "roundish" relics, in particular given its small LLS.

Based on the morphology of the source and the fact that it possesses all the trademark characteristics of a radio relic, we suggest that this is likely a new "roundish" relic. Note that for a "roundish" relic the previous calculation of the shock Mach number is not valid. However, we currently lack the required high-frequency observations to determine whether the integrated spectrum of the emission is curved, which is the predominant marker for a roundish relic. We therefore currently refrain from concluding that this is indeed a newly discovered radio relic.

\section{Conclusions}

In this work, we have presented new VLA radio observations of the cluster Abell 2744 at $1-4 \mathrm{GHz}$. Spectral index maps of the cluster, with resolutions ranging from $30^{\prime \prime}$ to $5^{\prime \prime}$, were constructed to study the origin of the diffuse emission in the cluster. In our images, we detect the previously known radio relic $\mathrm{R} 1$ and radio halo, along with three new diffuse sources, R2, R3, and R4. Below, we summarize our results.

1. The spectrum of the radio halo is reasonably well described by a single power law, with an integrated spectral index of $\alpha_{325}^{3000}=-1.32 \pm 0.14$, though there is some evidence of a potential steepening at higher frequencies. The spectral index maps reveal the spectral index across the radio halo to be rather uniform, although some steepening seems to occur at the faint outer parts of the halo. We do not find strong evidence for the presence of a correlation between spectral index and ICM temperature, as was suggested earlier for the radio halo by Orrú et al. (2007). The SE boundary of the radio halo is relatively well defined and aligns with the southern shock reported by Owers et al. (2011). Similar halo-shock edges have been observed in other well-studied clusters and this appears to be a more common phenomenon, possibly providing important information for our understanding of the formation of radio halos.

2. For the main relic R1, we measure an integrated spectral index of $\alpha=-1.32 \pm 0.09$, which is consistent with previous measurements. We observe a spectral index gradient across the relic's width, which is indicative of acceleration in an outward traveling shock, with radiative losses in the region downstream of the shock. We estimate a spectral index for relic injection of $\alpha_{\text {inj }}=-1.12 \pm 0.19$ from a spectral profile across the relic's width, corresponding to a shock Mach number of $\mathcal{M}=2.05_{-0.19}^{+0.31}$ under the assumption of DSA. This value is found to agree within the uncertainties with the X-ray Mach number derived by Eckert et al. (2016). Relic R1 is polarized at the $27 \%$ level, with the $E$-vectors 
perpendicularly oriented along the relic's major axis. Using this polarization fraction, we determine the viewing angle of relic $\mathrm{R} 1$ to be $\theta \gtrsim 50^{\circ}$, from which we constrain the geometry of the primary NE-SW merger axis to be within $\sim \pm 40^{\circ}$ of the plane of the sky.

3. The newly discovered relic R2 is located to the SE of the cluster core. Relic R2 is a $\sim 1.2 \mathrm{Mpc}$ long elongated source with an integrated spectral index of $\alpha=$ $-1.81 \pm 0.26$. The source is polarized at the $43 \%$ level, with the $E$-vectors perpendicular to the relic's orientation. From a Chandra X-ray image, we find evidence for a possible density jump at the relic's location. Fitting a broken power-law density model, we determine a density jump of $R=1.39_{-0.22}^{+0.34}$, which would correspond to a Mach number of $\mathcal{M}=1.26_{-0.15}^{+0.25}$. Using the mean polarization fraction we derive a relic viewing angle of $\sim 70^{\circ}$, corresponding to a merger axis within $\sim \pm 20^{\circ}$ of the plane of the sky.

4. The new relic R3 is located to the NW of the cluster and measures $\gtrsim 1.1 \mathrm{Mpc}$ in length. This relic is also highly polarized at the $\sim 30 \%$ level. For the integrated spectral index, we measure a value of $\alpha=-0.63 \pm 0.21$. The measured spectral index value of -0.63 is one of the flattest recorded for radio relics. Assuming what we measure is the injection spectral index, this would correspond to a high shock Mach number of $\mathcal{M}=4.04 \pm 1.4$ (under the assumption of DSA). Using the mean polarization fraction, we obtain a viewing angle of $\sim 52^{\circ}$. The orientation of the relic is peculiar, because it points northwest, away from the northwest subcluster.

5. We found a small patch of diffuse emission $\sim 200 \mathrm{kpc}$ north of the radio halo. The source, R4, measures $50 \times 30 \mathrm{kpc}^{2}$ in size. The spectral index maps reveal a spectral index gradient across R4 and it has an integrated spectral index value of $\alpha=-1.34 \pm 0.23$. The source is also highly polarized with a mean polarization fraction of $\sim 30 \%$. The source could be a radio phoenix or AGN relic given its small size.

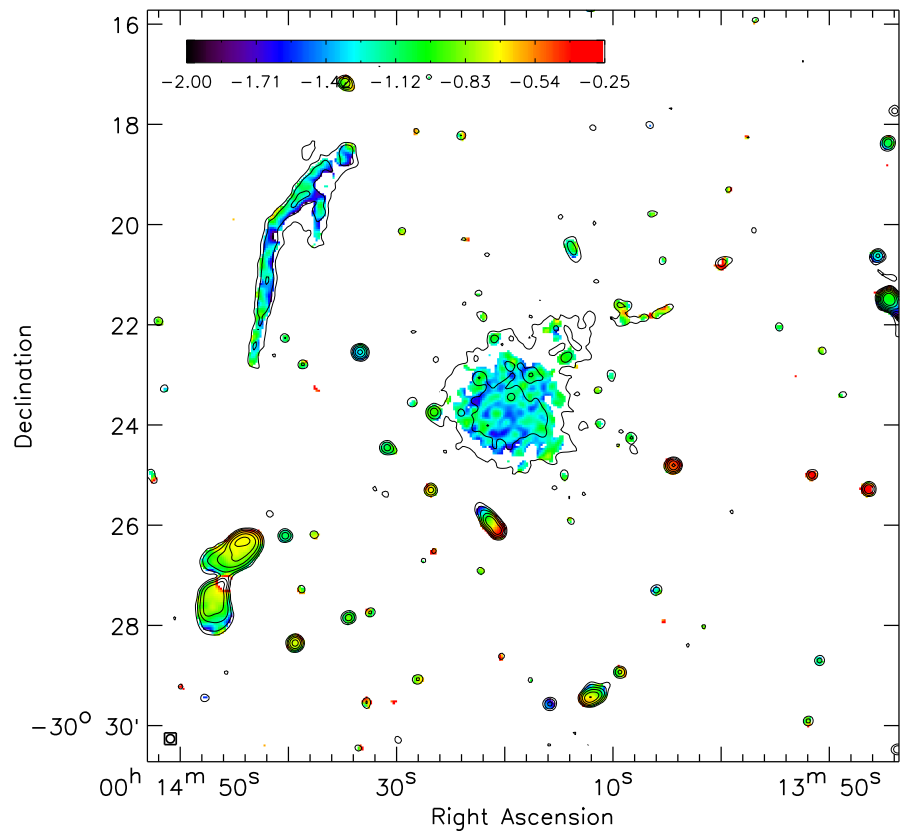

6. The detection of two new large relics adds yet more complexity to the already complicated merger history of A2744. The location and orientation of the new relic R2 could suggest that it traces a shock wave produced when the main cluster core collided with the northwestern interloper in a SE-NW event. The discovery of relic R3 seems to lend credence to a minor off-axis merger event between two subhalos suggested by Medezinski et al. (2016), although its precise location and orientation remain puzzling.

We thank the anonymous referee for useful comments. The National Radio Astronomy Observatory is a facility of the National Science Foundation operated under cooperative agreement by Associated Universities, Inc. Support for this work was provided by the National Aeronautics and Space Administration through Chandra Award Number GO516133X issued by the Chandra X-ray Observatory Center, which is operated by the Smithsonian Astrophysical Observatory for and on behalf of the National Aeronautics and Space Administration under contract NAS8-03060.

R.J.W. is supported by a Clay Fellowship awarded by the Harvard-Smithsonian Center for Astrophysics. W.R.F., C.J., and F.A.-S. acknowledge support from the Smithsonian Institution. F.A.-S. acknowledges support from Chandra grant GO3-14131X. Basic research in radio astronomy at NRL by TEC is supported by 6.1 Base funding. M.N. acknowledges PRIN-INAF 2014 1.05.01.94.02. This research made use of APLpy, an open-source plotting package for Python (Robitaille $\&$ Bressert 2012). This research made use of Astropy, a community-developed core Python package for Astronomy (Astropy Collaboration et al. 2013).

\section{Appendix A Spectral Index Uncertainty Maps, Region Locations, and T-T Plots}

Figure 21 in this appendix shows the 10 and 5 arcsecond resolution spectral index maps described in Section 4.4. The

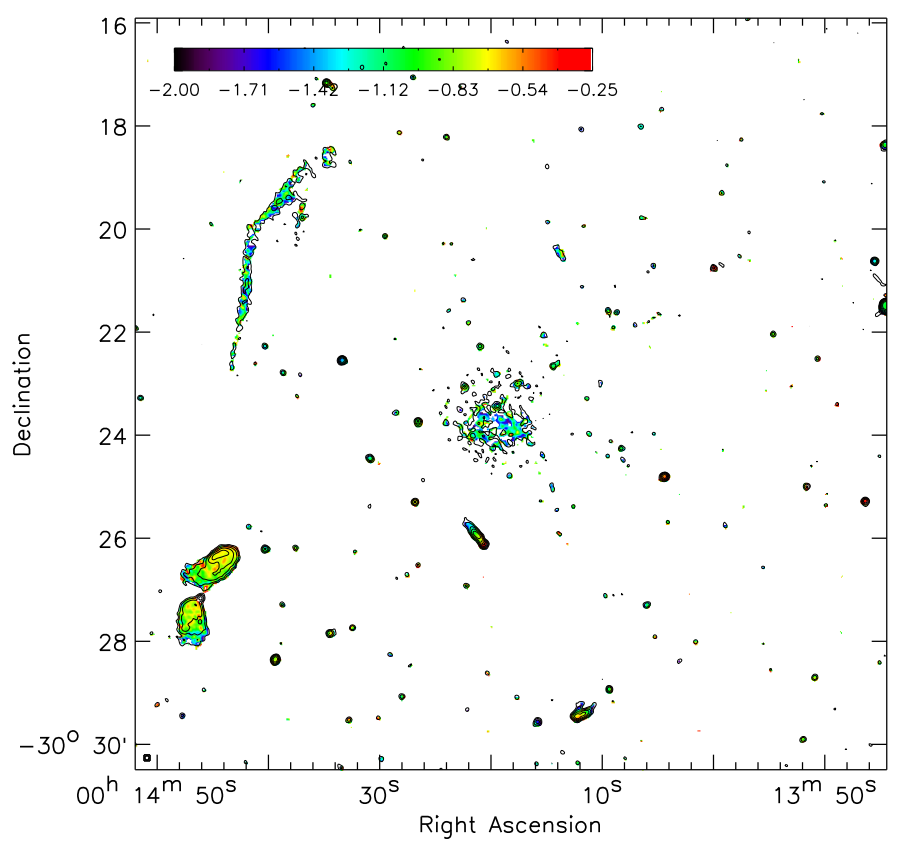

Figure 21. Spectral index maps of A2744 between 1.5 and $3.0 \mathrm{GHz}$, tapered to resolutions of $10^{\prime \prime}$ (left) and $5^{\prime \prime}$ (right). Contour levels are obtained from the $1.5 \mathrm{GHz}$ image and placed at the $[1,2,4,8, \ldots] \times 4 \sigma_{\text {rms }}$ and $3 \sigma_{\text {rms }}$ levels, respectively. 

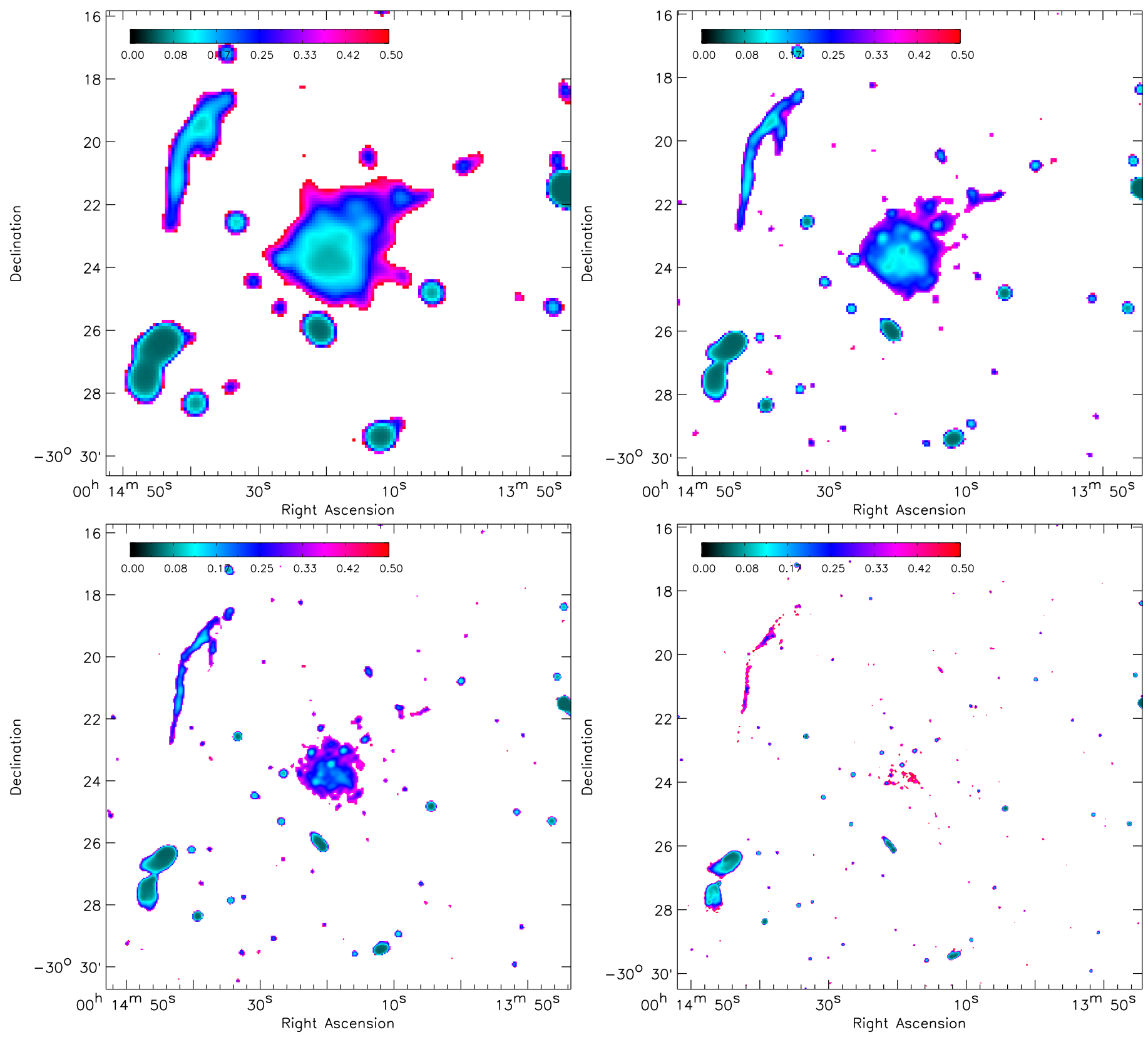

Figure 22. Spectral index error maps corresponding to Figures 8 (top) and 21 (bottom).

corresponding error maps for all resolutions are displayed in Figure 22. Figure 23 shows the regions used for the spectral index distribution analysis carried out for the radio halo in Section 5.1.1. The image displayed in Figure 24 shows the regions used to extract flux densities for the spectral index gradient analysis in Section 5.2.1. Finally, Figure 25 shows the results of T-T analyses carried out in both Sections 5.1.1 and 5.2.1. 

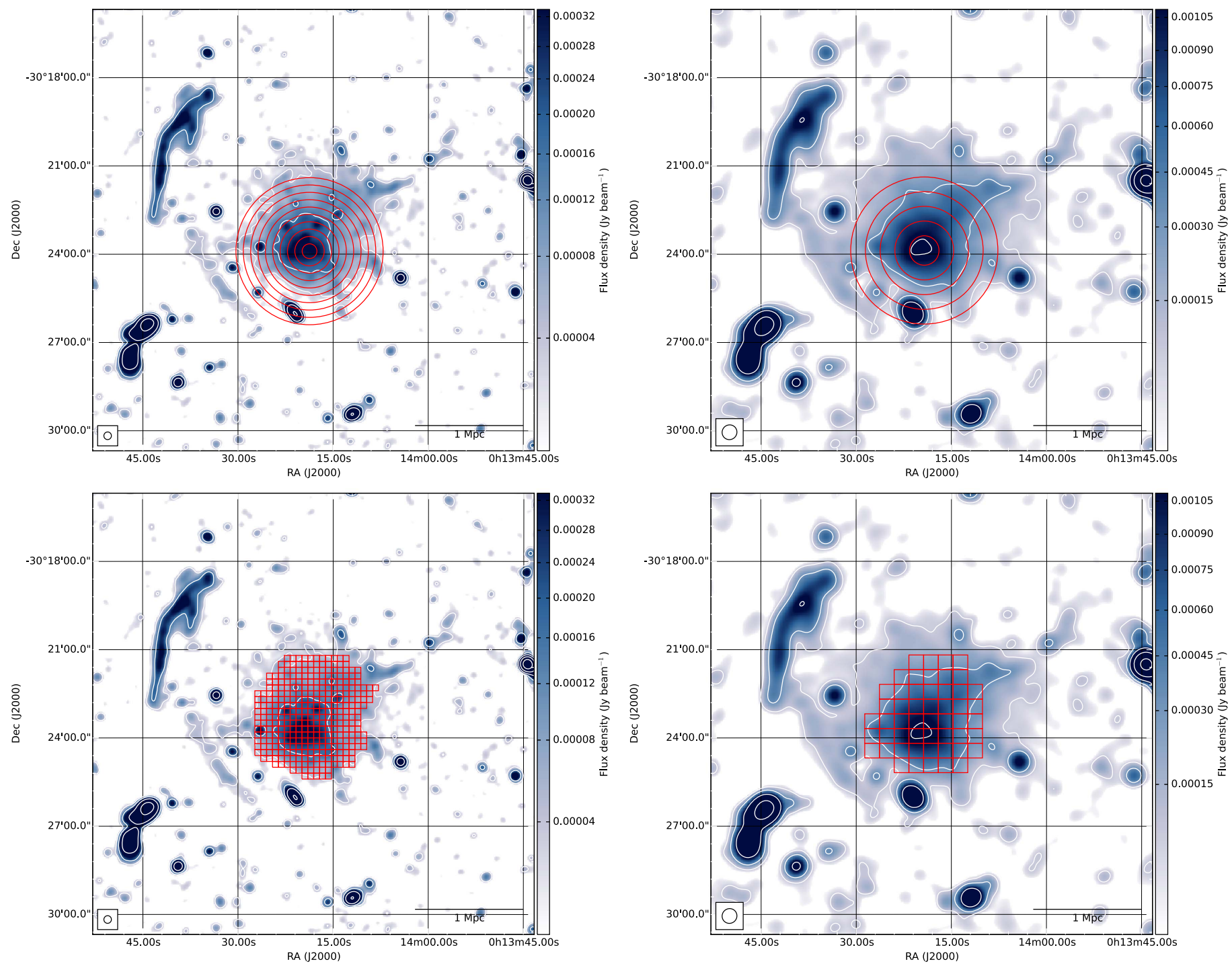

Figure 23. Regions where the spectral indices were extracted. Top: annuli to compute the radial profile of the spectral index of the halo, see Figure 11. These annuli have widths of $15^{\prime \prime}$ (left) and $30^{\prime \prime}$ (right) and are overlaid on the L + S-band combined images tapered to resolutions of $15^{\prime \prime}$ and $30^{\prime \prime}$. Bottom: the same images as in the top panel, but in this case boxes are shown in which we extracted the spectral indices, see Figure 10. 


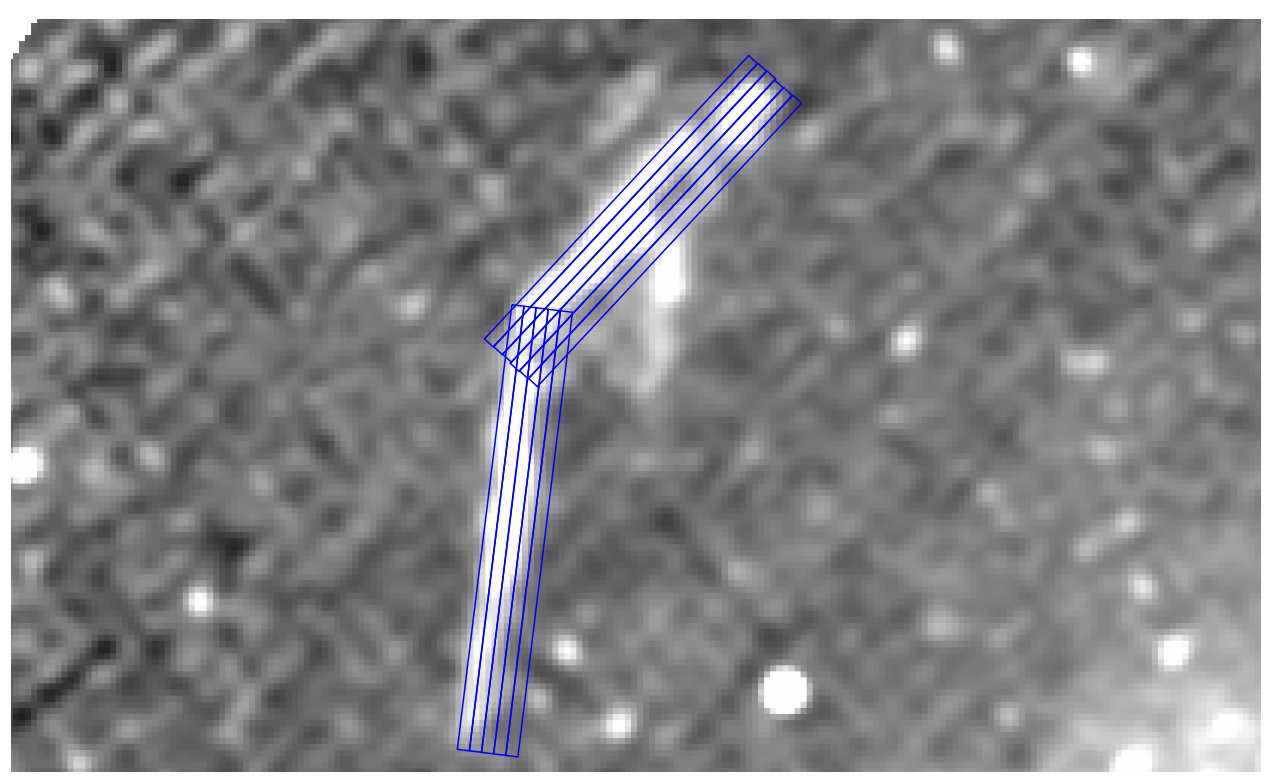

Figure 24. Image showing the $5^{\prime \prime}$ wide regions used to extract the spectral index gradient across the relic. In grayscale is the S-band $10^{\prime \prime}$ resolution image.
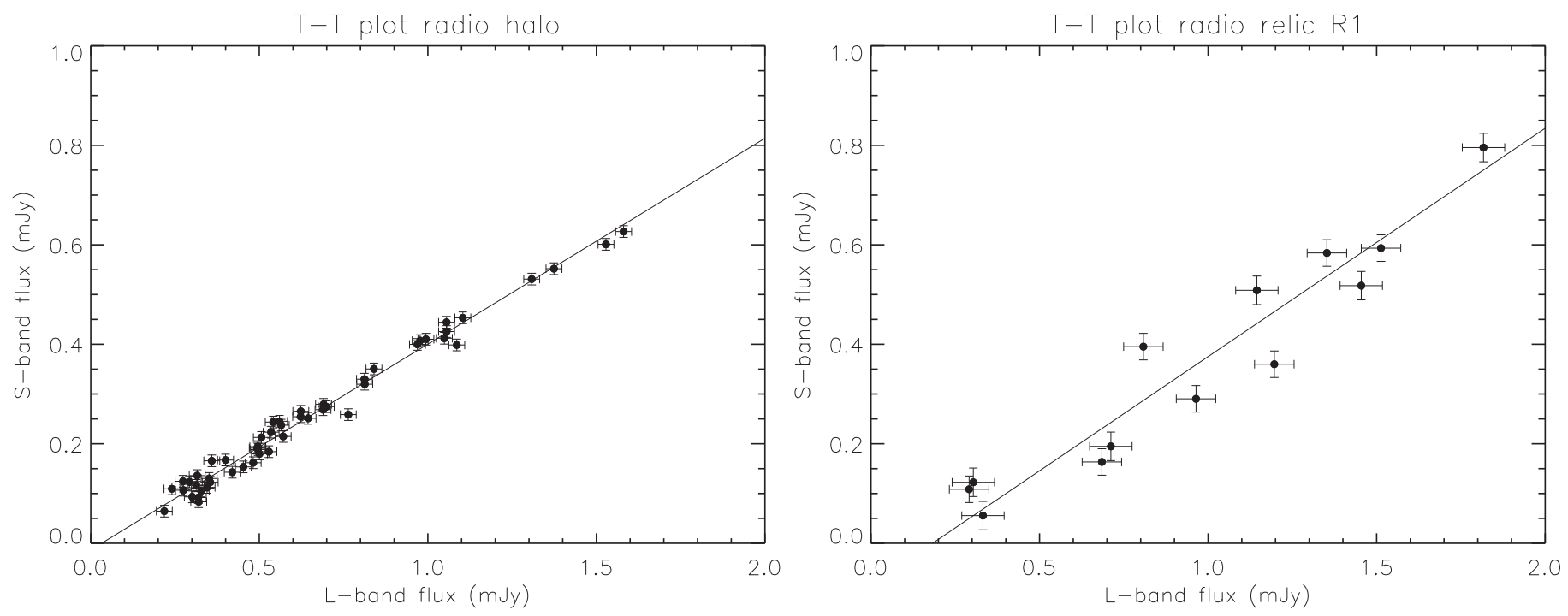

Figure 25. T-T plots (Turtle et al. 1962) for the radio halo (left) and relic (right), see Sections 5.1.1 and 5.2.1 for more details. 


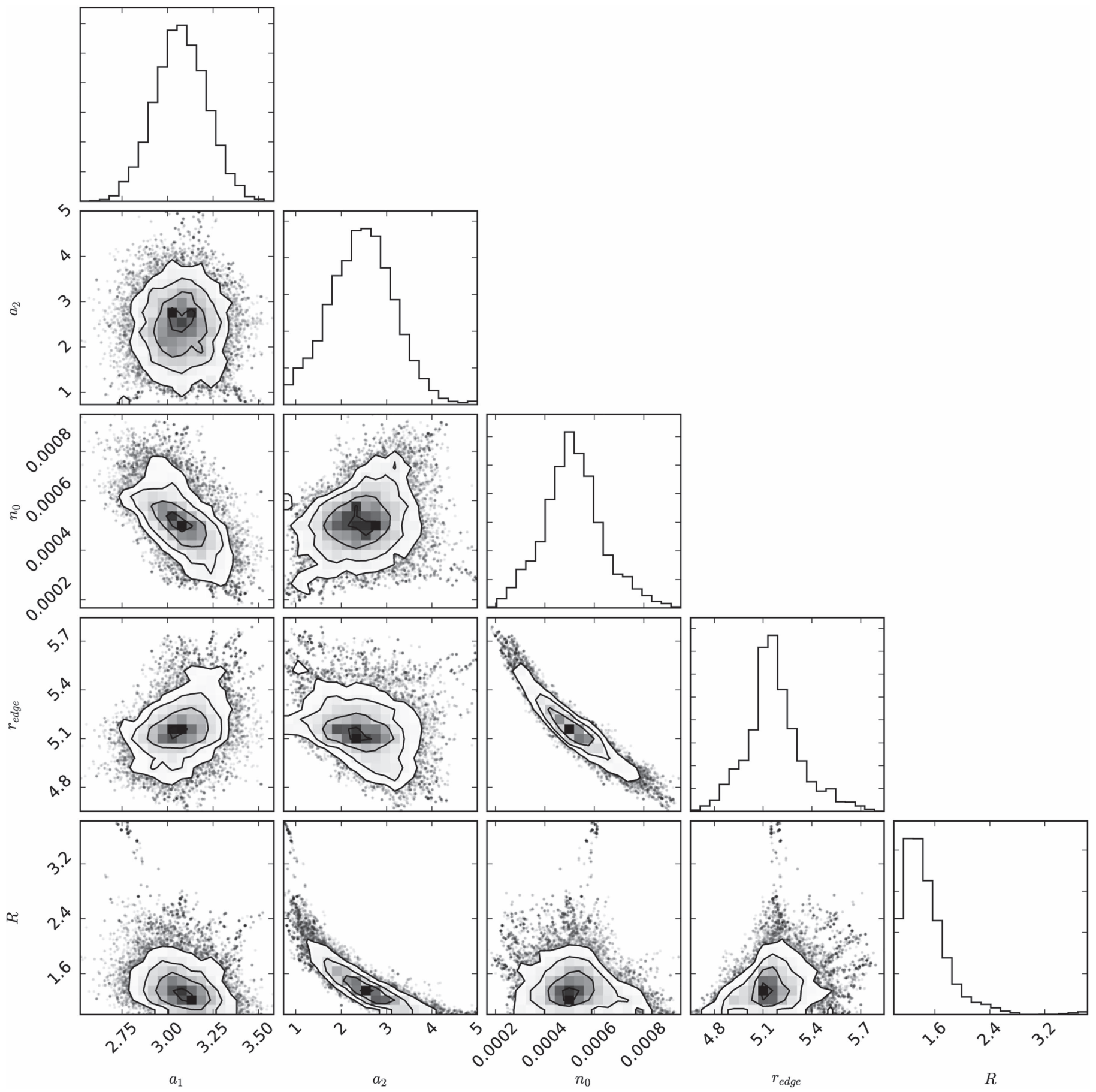

Figure 26. The MCMC "corner plot" (Foreman-Mackey 2016, 2017) for the distribution of the uncertainties in the fitted parameters for the X-ray surface brightness profile across relic R2 (see Section 5.2.2 and Equation (11)). Contour levels are drawn at $[0.5,1.0,1.5,2.0] \sigma$.

\section{Appendix B MCMC Corner Plot}

Figure 26 in this appendix shows the Markov Chain Monte Carlo "corner plot" for the distribution of the uncertainties in the fitted parameters (given in Equation (11)) for the X-ray surface brightness profile across relic R2. See Section 5.2.2 for details.

\section{ORCID iDs}

R. J. van Weeren (1) https://orcid.org/0000-0002-0587-1660

F. Andrade-Santos (i) https://orcid.org/0000-0002-8144-9285

W. R. Forman (1) https://orcid.org/0000-0002-9478-1682

T. E. Clarke (1) https://orcid.org/0000-0001-6812-7938

R. P. Kraft (1) https://orcid.org/0000-0002-0765-0511

E. Medezinski (1) https://orcid.org/0000-0001-7007-2358 
T. Mroczkowski (1) https://orcid.org/0000-0003-3816-5372

P. E. J. Nulsen (1) https://orcid.org/0000-0003-0297-4493

S. W. Randall 1 h https://orcid.org/0000-0002-3984-4337

K. Umetsu (1) https://orcid.org/0000-0002-7196-4822

\section{References}

Anders, E., \& Grevesse, N. 1989, GeCoA, 53, 197

Arnaud, K. A. 1996, in ASP Conf. Ser. 101, Astronomical Data Analysis Software and Systems V, ed. G. H. Jacoby \& J. Barnes (San Francisco, CA: ASP), 17

Astropy Collaboration, Robitaille, T. P., Tollerud, E. J., et al. 2013, A\&A, 558, A33

Bennett, C. L., Larson, D., Weiland, J. L., \& Hinshaw, G. 2014, ApJ, 794, 135

Bonafede, A., Brüggen, M., van Weeren, R., et al. 2012, MNRAS, 426, 40

Bonafede, A., Giovannini, G., Feretti, L., Govoni, F., \& Murgia, M. 2009, A\&A, 494, 429

Bonafede, A., Intema, H. T., Brüggen, M., et al. 2014, ApJ, 785, 1

Boschin, W., Girardi, M., Spolaor, M., \& Barrena, R. 2006, A\&A, 449, 461

Botteon, A., Gastaldello, F., Brunetti, G., \& Dallacasa, D. 2016, MNRAS, 460, L84

Braglia, F. G., Pierini, D., Biviano, A., \& Böhringer, H. 2009, A\&A, 500, 947

Briggs, D. S. 1995, PhD thesis, New Mexico Institute of Mining Technology, Socorro, NM, USA

Brunetti, G., \& Jones, T. W. 2014, IJMPD, 23, 30007

Brunetti, G., \& Lazarian, A. 2007, MNRAS, 378, 245

Brunetti, G., Setti, G., Feretti, L., \& Giovannini, G. 2001, MNRAS, 320, 365

Cassano, R. 2010, A\&A, 517, A10

Clarke, T. E., \& Enßlin, T. A. 2006, AJ, 131, 2900

Colless, M., Peterson, B. A., Jackson, C., et al. 2003, arXiv:astro-ph/0306581

Cornwell, T. J. 2008, ISTSP, 2, 793

Cortese, L., Marcillac, D., Richard, J., et al. 2007, in IAU Symp. 235, Galaxy Evolution across the Hubble Time, ed. F. Combes \& J. Palouš (Cambridge: Cambridge Univ. Press), 198

de Gasperin, F., Ogrean, G. A., van Weeren, R. J., et al. 2015, MNRAS, 448, 2197

Dennison, B. 1980, ApJL, 239, L93

Drury, L. O. 1983, RPPh, 46, 973

Ebeling, H., Stephenson, L. N., \& Edge, A. C. 2014, ApJL, 781, L40

Eckert, D., Jauzac, M., Vazza, F., et al. 2016, arXiv:1603.02272

Enßlin, T. A., Biermann, P. L., Klein, U., \& Kohle, S. 1998, A\&A, 332, 395

Enßlin, T. A., \& Gopal-Krishna 2001, A\&A, 366, 26

Feretti, L., Giovannini, G., Govoni, F., \& Murgia, M. 2012, A\&ARv, 20, 54

Feretti, L., Orrù, E., Brunetti, G., et al. 2004, A\&A, 423, 111

Ferrari, C., Govoni, F., Schindler, S., Bykov, A. M., \& Rephaeli, Y. 2008, SSRv, 134, 93

Foreman-Mackey, D. 2016, corner.py: Scatterplot matrices in Python, The Journal of Open Source Software, 24, doi:10.21105/joss.00024

Foreman-Mackey, D. 2017, corner: Corner plots, Astrophysics Source Code Library, ascl: 1702.002

Foreman-Mackey, D., Hogg, D. W., Lang, D., \& Goodman, J. 2013, PASP, 125,306

Fujita, Y., Takizawa, M., Yamazaki, R., Akamatsu, H., \& Ohno, H. 2015, ApJ, 815,116

Giacintucci, S., Venturi, T., Macario, G., et al. 2008, A\&A, 486, 347

Giovannini, G., Feretti, L., \& Stanghellini, C. 1991, A\&A, 252, 528

Giovannini, G., Tordi, M., \& Feretti, L. 1999, NewA, 4, 141

Govoni, F., Feretti, L., Giovannini, G., et al. 2001, A\&A, 376, 803

Hindson, L., Johnston-Hollitt, M., Hurley-Walker, N., et al. 2014, MNRAS, 445,330

Ibaraki, Y., Ota, N., Akamatsu, H., Zhang, Y.-Y., \& Finoguenov, A. 2014, A\&A, 562, A11

Jaffe, W. J. 1977, ApJ, 212, 1

Jauzac, M., Eckert, D., Schwinn, J., et al. 2016, MNRAS, 463, 3876

Kalberla, P. M. W., Burton, W. B., Hartmann, D., et al. 2005, A\&A, 440, 775

Kang, H. 2015, JKAS, 48, 9

Kang, H., \& Ryu, D. 2011, ApJ, 734, 18

Kang, H., \& Ryu, D. 2015, ApJ, 809, 186
Kempner, J. C., Blanton, E. L., Clarke, T. E., et al. 2004, in The Riddle of Cooling Flows in Galaxies and Clusters of Galaxies, ed. T. Reiprich, J. Kempner, \& N. Soker (http://www.astro.virginia.edu/coolflow/) 335

Kempner, J. C., \& David, L. P. 2004, MNRAS, 349, 385

Landau, L. D., \& Lifshitz, E. M. 1959, in Fluid Mechanics, ed. L. D. Landau \& E. M. Lifshitz (London: Pergamon)

Lotz, J., Mountain, M., Grogin, N. A., et al. 2014, American Astronomical Society Meeting Abstracts, 223, 254.01

Lotz, J. M., Koekemoer, A., Coe, D., et al. 2017, ApJ, 837, 97

Macario, G., Markevitch, M., Giacintucci, S., et al. 2011, ApJ, 728, 82

Markevitch, M. 2010, arXiv:1010.3660

Markevitch, M., Gonzalez, A. H., David, L., et al. 2002, ApJL, 567, L27

Markevitch, M., Govoni, F., Brunetti, G., \& Jerius, D. 2005, ApJ, 627, 733

Markwardt, C. B. 2009, in ASP Conf. Ser. 411, Astronomical Data Analysis Software and Systems XVIII, ed. D. A. Bohlender, D. Durand, \& P. Dowler (San Francisco, CA: ASP), 251

McMullin, J. P., Waters, B., Schiebel, D., Young, W., \& Golap, K. 2007, in ASP Conf. Ser. 376, Astronomical Data Analysis Software and Systems XVI, ed. R. A. Shaw, F. Hill, \& D. J. Bell (San Francisco, CA: ASP), 127

Medezinski, E., Umetsu, K., Okabe, N., et al. 2016, ApJ, 817, 24

Merten, J., Coe, D., Dupke, R., et al. 2011, MNRAS, 417, 333

Mohan, N., \& Rafferty, D. 2015, PyBDSM: Python Blob Detection and Source Measurement, Astrophysics Source Code Library, ascl:1502.007

Murgia, M., Govoni, F., Markevitch, M., et al. 2009, A\&A, 499, 679

Offringa, A. R., McKinley, B., Hurley-Walker, N., et al. 2014, MNRAS, 444,606

Ogrean, G. 2016, in American Astronomical Society Meeting Abstracts, $228,317.09$

Ogrean, G. 2017, in American Astronomical Society Meeting Abstracts, $229,438.08$

Orrú, E., Murgia, M., Feretti, L., et al. 2007, A\&A, 467, 943

Owers, M. S., Couch, W. J., Nulsen, P. E. J., \& Randall, S. W. 2012, ApJL, 750, L23

Owers, M. S., Randall, S. W., Nulsen, P. E. J., et al. 2011, ApJ, 728, 27

Perley, R. A., \& Butler, B. J. 2013, ApJS, 204, 19

Petrosian, V. 2001, ApJ, 557, 560

Pinzke, A., Oh, S. P., \& Pfrommer, C. 2013, MNRAS, 435, 1061

Planck Collaboration, Ade, P. A. R., Aghanim, N., et al. 2013, A\&A, 554, A140

Rau, U., \& Cornwell, T. J. 2011, A\&A, 532, A71

Robitaille, T., \& Bressert, E. 2012, APLpy: Astronomical Plotting Library in Python, Astrophysics Source Code Library, ascl:1208.017

Rybicki, G. B., \& Lightman, A. P. 1986, Radiative Processes in Astrophysics (New York: Wiley-VCH), 400

Sanders, J. S. 2006, MNRAS, 371, 829

Schlickeiser, R., Sievers, A., \& Thiemann, H. 1987, A\&A, 182, 21

Shimwell, T. W., Brown, S., Feain, I. J., et al. 2014, MNRAS, 440, 2901

Shimwell, T. W., Markevitch, M., Brown, S., et al. 2015, MNRAS, 449, 1486

Taylor, A. R., Stil, J. M., \& Sunstrum, C. 2009, ApJ, 702, 1230

Trasatti, M., Akamatsu, H., Lovisari, L., et al. 2015, A\&A, 575, A45

Turtle, A. J., Pugh, J. F., Kenderdine, S., \& Pauliny-Toth, I. I. K. 1962, MNRAS, 124, 297

Uchida, Y., Simionescu, A., Takahashi, T., et al. 2016, PASJ, 68, S20

Vacca, V., Feretti, L., Giovannini, G., et al. 2014, A\&A, 561, A52

van Weeren, R. J., Andrade-Santos, F., Dawson, W. A., et al. 2017a, NatAs, 1,0005

van Weeren, R. J., Brunetti, G., Brüggen, M., et al. 2016, ApJ, 818, 204

van Weeren, R. J., Ogrean, G. A., Jones, C., et al. 2017b, ApJ, 835, 197

van Weeren, R. J., Röttgering, H. J. A., \& Brüggen, M. 2011, A\&A, 527 A114

van Weeren, R. J., Röttgering, H. J. A., Brüggen, M., \& Hoeft, M. 2010, Sci, 330,347

van Weeren, R. J., Röttgering, H. J. A., Intema, H. T., et al. 2012a, A\&A, $546, \mathrm{~A} 124$

van Weeren, R. J., Röttgering, H. J. A., Rafferty, D. A., et al. 2012b, A\&A, 543, A43

Venturi, T., Giacintucci, S., Dallacasa, D., et al. 2013, A\&A, 551, A24

Vikhlinin, A., Markevitch, M., Murray, S. S., et al. 2005, ApJ, 628, 655

Williams, M. J., Bureau, M., \& Cappellari, M. 2010, MNRAS, 409, 1330 